\title{
A method to optimize selection on multiple identified quantitative trait loci
}

\author{
Reena Chakraborty ${ }^{\mathrm{a}}$, Laurence Moreau ${ }^{\mathrm{b}}$, \\ Jack C.M. DEKKERS ${ }^{\text {a* }}$ \\ a Department of Animal Science, 225C Kildee Hall, \\ Iowa State University Ames, IA, 50011, USA \\ b InRA-UPS-INA PG, Station de génétique végétale, \\ Ferme du Moulon, 91190 Gif-sur-Yvette, France \\ (Received 5 February 2001; accepted 15 October 2001)
}

\begin{abstract}
A mathematical approach was developed to model and optimize selection on multiple known quantitative trait loci (QTL) and polygenic estimated breeding values in order to maximize a weighted sum of responses to selection over multiple generations. The model allows for linkage between QTL with multiple alleles and arbitrary genetic effects, including dominance, epistasis, and gametic imprinting. Gametic phase disequilibrium between the QTL and between the QTL and polygenes is modeled but polygenic variance is assumed constant. Breeding programs with discrete generations, differential selection of males and females and random mating of selected parents are modeled. Polygenic EBV obtained from best linear unbiased prediction models can be accommodated. The problem was formulated as a multiplestage optimal control problem and an iterative approach was developed for its solution. The method can be used to develop and evaluate optimal strategies for selection on multiple QTL for a wide range of situations and genetic models.
\end{abstract}

\section{selection / quantitative trait loci / optimization / marker assisted selection}

\section{INTRODUCTION}

In the past decades, several genes with substantial effects on quantitative traits have been identified, facilitated by developments in molecular genetics. Prime examples in pigs are the ryanodine receptor gene for stress susceptibility and meat quality [8] and the estrogen receptor gene for litter size [17]. Parallel efforts in the search for genes that affect quantitative traits have focused on the identification of genetic markers that are linked to quantitative trait loci (QTL) $[1,9]$. In the remainder of this paper, QTL for which the causative mutation or a tightly linked marker with strong linkage disequilibrium across the population has been identified, will be referred to as an identified QTL, in

\footnotetext{
* Correspondence and reprints E-mail: jdekkers@iastate.edu
} 
contrast to a marked QTL, for which a marker is available that is in linkage equilibrium with the QTL.

Strategies for the use of identified or marked QTL in selection have generally focused on selecting individuals for breeding based on the following index [19]: $I=\alpha+\widehat{B V}$, where $\alpha$ is an estimate of the breeding value of the individual for the identified or marked QTL and $\widehat{B V}$ is an estimate of the polygenic effect of the individual, which includes the collective effect of all other genes and is estimated from the phenotype. This selection strategy will be referred to as standard QTL selection in the remainder of this paper. Advanced statistical methodology based on best linear unbiased prediction (BLUP) has been developed to estimate the components of this index ( $\alpha$ and $\widehat{B V}$ ), using all available genotypic and phenotypic data for either marked [7] or identified QTL [12].

Gibson [10] investigated the longer term consequences of standard QTL selection on an identified QTL using computer simulation, and showed that, although such selection increases selection response in the short term, it can result in lower response in the longer term than selection without QTL information (phenotypic selection). These results, which have been confirmed by several authors $[13,16]$, show that, although standard QTL selection increases the frequency of the QTL in the short term, this is at the expense of response in polygenic breeding values. Because of the non-linear relationship between selected proportion and selection intensity, polygenic response lost in early generations is never entirely regained in later generations [5]. The end result is a lower genetic level for standard QTL selection than phenotypic selection when the identified gene is fixed for both selection strategies. The lower longer-term response results from suboptimal use of QTL information in selection.

Dekkers and van Arendonk [5] developed a model to optimize selection on an identified QTL over multiple generations. Optimal strategies were derived by formulating the optimization problem as an optimal control problem [14]. This allowed for the development of an efficient strategy for solving the optimization problem. Manfredi et al. [15] used a sequential quadratic programming package to optimize selection and mating with an identified QTL for a sex-limited trait as a general constrained non-linear programming problem. Although their method allows for greater flexibility with regard to structure of the breeding program, including overlapping generations and non-random mating, computational requirements are much greater than for the optimal control approach, which capitalizes on the recursive nature of genetic improvement over multiple generations.

The model of Dekkers and van Arendonk [5] was restricted to equal selection among males and females, a single identified QTL with additive effects, and optimization of cumulative response in the final generation of a planning horizon. These assumptions are too restrictive for applications to practical breeding programs. With multiple QTL identified in practical breeding programs, there 
is in particular a lack of methodology to derive strategies for optimal selection on multiple QTL, as pointed out by Hospital et al. [11]. Nor is the methodology available for selection on QTL with non-additive effects, including epistasis and gametic imprinting. Therefore, the objective of this study was to extend the method of Dekkers and van Arendonk [5] to selection programs with different selection strategies for males and females, maximizing a weighted combination of short and longer-term responses, and to multiple identified QTL, allowing for non-additive effects at the QTL, including dominance, epistasis and gametic imprinting. The method derived here was applied to optimizing selection on two linked QTL in a companion paper [4].

\section{METHODS}

We first describe the deterministic model for selection on one QTL with two alleles and dominance and differential selection in males and females, extending the method of Dekkers and van Arendonk [5]. Where possible, the notation established in Dekkers and van Arendonk [5] is followed. The equations are developed in vector notation, which allows subsequent generalization to multiple QTL.

\subsection{Model for a single QTL with two alleles}

Consider selection in an outbred population with discrete generations for a quantitative trait that is affected by an identified QTL with two alleles (B and b), additive polygenic effects that conform to the infinitesimal genetic model [6], and normally distributed environmental effects. Effects at the QTL are assumed known without error and all individuals are genotyped for the QTL prior to selection. Sires and dams which are to produce the next generation are selected on a combination of their QTL genotype and an estimated breeding value (EBV) for polygenic effects. Conceptually, polygenic EBV can be estimated from a BLUP model that includes the QTL as a fixed or random effect, using information from all relatives. Selected sires and dams are mated at random. The model accounts for the gametic phase disequilibrium [2] between the QTL and polygenes that is induced by selection but polygenic variance is assumed constant.

\subsubsection{Variables and notation}

The variables for the deterministic model are defined below and are summarized in Table I. They are indexed by $\operatorname{sex} j, j=\mathrm{s}$ for males and $j=\mathrm{d}$ for females, QTL allele or genotype number $k$, and generation $t$. The allele index, $k$, is 1 for allele $\mathrm{B}$ and 2 for allele $\mathrm{b}$. When indexed by genotype, $k=1,2$, 3 , and 4 for genotypes $\mathrm{BB}, \mathrm{Bb}, \mathrm{bB}$, and $\mathrm{bb}$, respectively, where the first letter indicates the allele received from the sire. The generation index, $t$, runs from 
Table I. Notation for genotype frequencies, fractions selected, proportions of B and b gametes produced by each genotype, mean polygenic breeding values, and selection differentials for sires of each genotype in generation $t$.

\begin{tabular}{|c|c|c|c|c|c|c|c|c|}
\hline \multirow[t]{2}{*}{ Genotype } & \multirow[t]{2}{*}{$\begin{array}{l}\text { Index } \\
\text { number }\end{array}$} & \multirow[t]{2}{*}{$\begin{array}{l}\text { Genotype } \\
\text { Frequency }\end{array}$} & \multirow[t]{2}{*}{$\begin{array}{l}\text { Fraction } \\
\text { Selected }\end{array}$} & \multicolumn{2}{|c|}{$\begin{array}{c}\text { Proportion of alleles } \\
\text { produced }\end{array}$} & \multirow[t]{2}{*}{$\begin{array}{l}\text { QTL } \\
\text { effect }\end{array}$} & \multirow[t]{2}{*}{$\begin{array}{l}\text { Mean polygenic } \\
\text { breeding value }\end{array}$} & \multirow[t]{2}{*}{$\begin{array}{l}\text { Selection } \\
\text { differential }\end{array}$} \\
\hline & & & & $\mathrm{B}$ & $\mathrm{b}$ & & & \\
\hline $\mathrm{BB}$ & 1 & $p_{\mathrm{s}, 1, t} p_{\mathrm{d}, 1, t}$ & $f_{\mathrm{s}, 1, t}$ & 1 & 0 & $a$ & $A_{\mathrm{s}, 1, t}+A_{\mathrm{d}, 1, t}$ & $S_{\mathrm{s}, 1, t}$ \\
\hline $\mathrm{Bb}$ & 2 & $p_{\mathrm{s}, 1, t} p_{\mathrm{d}, 2, t}$ & $f_{\mathrm{s}, 2, t}$ & $1 / 2$ & $1 / 2$ & $d$ & $A_{\mathrm{s}, 1, t}+A_{\mathrm{d}, 2, t}$ & $S_{\mathrm{s}, 2, t}$ \\
\hline $\mathrm{bB}$ & 3 & $p_{\mathrm{s}, 2, t} p_{\mathrm{d}, 1, t}$ & $f_{\mathrm{s}, 3, t}$ & $1 / 2$ & $1 / 2$ & $d$ & $A_{\mathrm{s}, 2, t}+A_{\mathrm{d}, 1, t}$ & $S_{\mathrm{s}, 3, t}$ \\
\hline $\mathrm{bb}$ & 4 & $p_{\mathrm{s}, 2, t} p_{\mathrm{d}, 2, t}$ & $f_{\mathrm{s}, 4, t}$ & 0 & 1 & $-a$ & $A_{\mathrm{s}, 2, t}+A_{\mathrm{d}, 2, t}$ & $S_{\mathrm{s}, 4, t}$ \\
\hline \multicolumn{2}{|c|}{ Vector notation } & $v_{t}$ & $f_{\mathrm{s}, t}$ & $n_{1}$ & $n_{2}$ & $q$ & $B V_{t}$ & $\boldsymbol{S}_{\mathrm{s}, t}$ \\
\hline
\end{tabular}


$t=0$ for the foundation generation to $t=T$ for the terminal generation of the planning horizon.

Let $p_{\mathrm{s}, 1, t}$ and $p_{\mathrm{s}, 2, t}$ denote the frequencies of alleles $\mathrm{B}$ and $\mathrm{b}$ at the identified QTL among paternal gametes that create generation $t$. Similarly, $p_{\mathrm{d}, 1, t}$ and $p_{\mathrm{d}, 2, t}$ are the allele frequencies among maternal gametes that create generation $t$. Note that $p_{\mathrm{s}, 2, t}=1-p_{\mathrm{s}, 1, t}$ but this relationship will not be used here to maintain the generality of the derivations. Vectors $\boldsymbol{p}_{j, t}$ for every $t=0, \ldots, T$, and $j=\mathrm{s}, \mathrm{d}$ are defined as

$$
\boldsymbol{p}_{j, t}=\left[p_{j, 1, t} p_{j, 2, t}\right]^{\prime} \text {. }
$$

Let $v_{k, t}$ be the frequency of the $k$ th QTL genotype in generation $t$. Under random mating, $v_{k, t}$ is the product of allele frequencies among paternal and maternal gametes, e.g., for genotype $\mathrm{Bb}, v_{2, t}=p_{\mathrm{s}, 1, t} p_{\mathrm{d}, 2, t}$. The $4 \times 1$ column vector $\boldsymbol{v}_{t}$ with components $v_{k, t}$ (Tab. I) is then computed as:

$$
\boldsymbol{v}_{t}=\boldsymbol{p}_{\mathrm{s}, t} \otimes \boldsymbol{p}_{\mathrm{d}, t}
$$

where $\otimes$ denotes the Kronecker product [18].

Let $q_{k}$ denote the genetic value of the QTL genotype $k$ and $\boldsymbol{q}$ the vector of the genetic values for all QTL genotypes. For a QTL with two alleles, $\boldsymbol{q}=[+a, d, d,-a]^{\prime}$, with $a$ the additive effect and $d$ the dominance effect [6].

Selection introduces gametic phase disequilibrium between the QTL and polygenes. With random mating of selected parents, this disequilibrium can be accounted for by modeling mean polygenic values by the type of gamete [5]. Denote the mean polygenic value of paternal and maternal gametes that carry allele $k$ and produce generation $t$ by $A_{\mathrm{s}, k, t}$ and $A_{\mathrm{d}, k, t}$, respectively. The mean polygenic value of individuals of, e.g., genotype $\mathrm{Bb}$ in generation $t$ is then $B V_{2, t}=A_{\mathrm{s}, 1, t}+A_{\mathrm{d}, 2, t}$. To obtain a vector representation of mean polygenic breeding values by genotype, $\boldsymbol{B} \boldsymbol{V}_{t}$, define vectors $\boldsymbol{A}_{j, t}$ for every $t=0, \ldots, T$ and $j=\mathrm{s}$, d as $\boldsymbol{A}_{j, t}=\left[A_{j, 1, t} A_{j, 2, t}\right]^{\prime}$, and $\boldsymbol{J}_{m}$ as an $m \times 1$ column vector with each element equal to one. Then,

$$
\boldsymbol{B} \boldsymbol{V}_{t}=\boldsymbol{A}_{\mathrm{s}, t} \otimes \boldsymbol{J}_{2}+\boldsymbol{J}_{2} \otimes \boldsymbol{A}_{\mathrm{d}, t} .
$$

The mean genetic value of the $k$ th genotype in generation $t, g_{k, t}$, is the sum of the value associated with the QTL genotype $k, q_{k}$, and the mean polygenic value $B V_{k, t}$. The genetic value vector $\boldsymbol{g}_{t}$ is the sum of $\boldsymbol{q}$ and $\boldsymbol{B} \boldsymbol{V}_{t}$ (Tab. I). The population mean genetic value in generation $t, G_{t}$, is the dot product of $\boldsymbol{v}_{t}^{\prime}$ and $\boldsymbol{g}_{t}$ :

$$
G_{t}=\boldsymbol{v}_{t}^{\prime} \boldsymbol{g}_{t} \text {. }
$$

\subsubsection{Selection model}

Selection is on an index of the identified QTL and the polygenic EBV. Following Dekkers and van Arendonk [5], such selection can be represented by truncation selection across four normal distributions for the polygenic EBV, with means equal to the index value for the QTL (Fig. 1). 


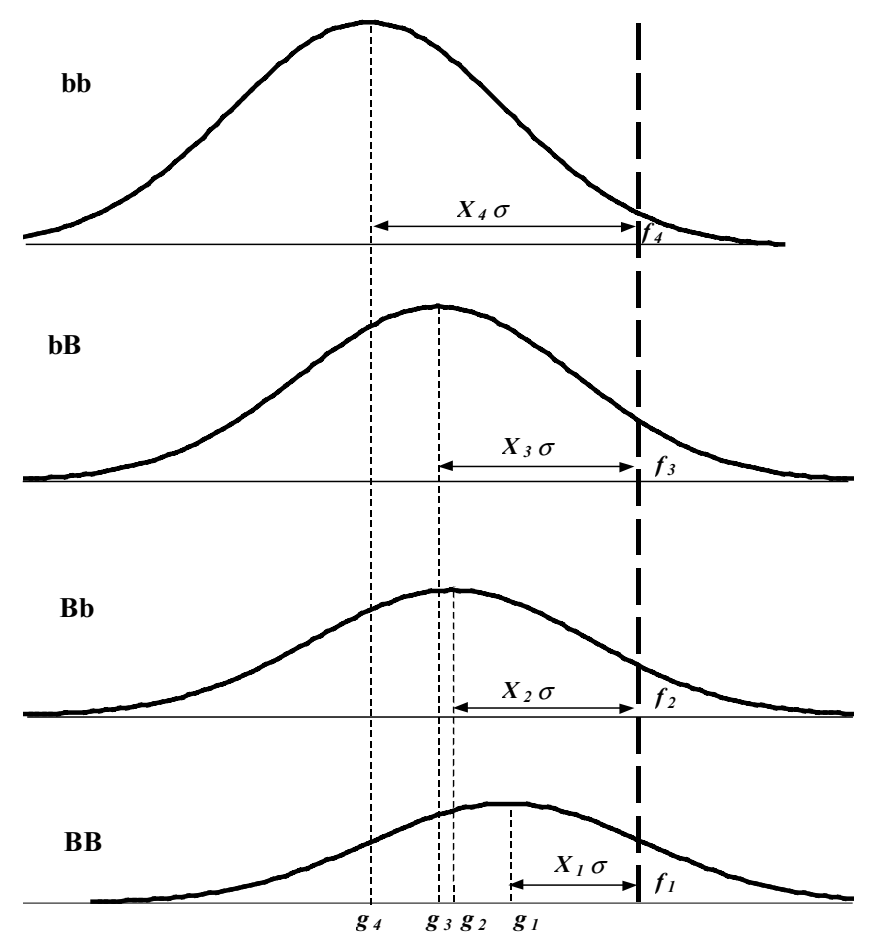

Figure 1. Representation of the process of selection on information from a QTL and estimates of polygenic breeding values. The QTL has two alleles (B and b). Estimates of polygenic breeding values have a standard deviation equal to $\sigma$. Selection is by truncation across four Normal distributions at a common truncation point on the index scale and, for the QTL genotype $k$, at standardized truncation points $X_{k}$ and with fraction selected $f_{k}$.

Let $Q_{\mathrm{s}}$ and $Q_{\mathrm{d}}$ be the fractions of males and females selected to produce the next generation as sires and dams, respectively. Let $f_{j, k, t}$ be the proportion of individuals of sex $j$ and genotype $k$ that is selected in generation $t$ (Tab. I) and $\boldsymbol{f}_{j, t}$ the corresponding vector of selected proportions. The total fraction of sires and dams selected in each generation across genotypes must equal the respective $Q_{j}$. Thus, for every $t=0, \ldots, T-1$ and $j=\mathrm{s}$, d:

$$
Q_{j}=\sum_{k=1}^{4} f_{j, k, t} v_{j, k, t}
$$

or

$$
Q_{j}-\boldsymbol{f}_{j, t}^{\prime} \boldsymbol{v}_{t}=0 .
$$

The frequency of, e.g., allele $B$ among paternal gametes that produce generation $t+1$, can then be computed as the sum of the fraction of $B$ gametes produced 
by genotype $k(0,1 / 2$, or 1 , see Tab. I) weighted by the relative frequency of genotype $k$ among the selected sires $\left(v_{j, k, t} f_{j, k, t} / Q_{j}\right)$ :

$$
p_{\mathrm{s}, 1, t+1}=\left(v_{\mathrm{s}, 1, t} f_{\mathrm{s}, 1, t}+1 / 2 v_{\mathrm{s}, 2, t} f_{\mathrm{s}, 2, t}+1 / 2 v_{\mathrm{s}, 3, t} f_{\mathrm{s}, 3, t}\right) / Q_{\mathrm{s}} .
$$

Similar equations are true for $p_{\mathrm{s}, 2, t+1}, p_{\mathrm{d}, 1, t+1}$ and $p_{\mathrm{d}, 2, t+1}$. To derive a vector representation of equation (7), let $\boldsymbol{N}$ be a matrix with columns corresponding to alleles and rows corresponding to genotypes and with element $N_{k, l}$ equal to the fraction of gametes with allele $l$ that is produced by genotype $k(0,1 / 2$, or 1$)$. Columns of matrix $\boldsymbol{N}\left(\boldsymbol{n}_{1}\right.$ and $\left.\boldsymbol{n}_{2}\right)$ are shown in Table I for the case of one QTL with two alleles. Then, for every $t=0, \ldots, T-1$, and $j=\mathrm{s}$, d,

$$
\boldsymbol{p}_{j, t+1}=\boldsymbol{N}^{\prime}\left(\boldsymbol{v}_{t} \circ \boldsymbol{f}_{j, t}\right) / Q_{j}
$$

where the symbol $\circ$ denotes the Hadamard product [18]. The vector of QTL allele frequencies in generation $t+1$ is:

$$
\boldsymbol{p}_{t+1}=1 / 2\left(\boldsymbol{p}_{\mathrm{s}, t+1}+\boldsymbol{p}_{\mathrm{d}, t+1}\right) .
$$

Following quantitative genetics selection theory [6], the mean polygenic breeding value of selected individuals of genotype $k$ in generation $t$ is:

$$
B V_{k, t}+S_{j, k, t}=B V_{k, t}+i_{j, k, t} \sigma_{j}
$$

where $S_{j, k, t}$ is the polygenic superiority of selected individuals, $i_{j, k, t}$ is the selection intensity associated with the selected fraction $f_{j, k, t}[6]$, and $\sigma_{j}$ is the standard deviation of estimates of polygenic breeding values for $\operatorname{sex} j$. Given the accuracy of estimated polygenic breeding values, $r_{j}$, and the polygenic standard deviation, $\sigma_{\mathrm{pol}}$, the standard deviation of polygenic EBV is $\sigma_{j}=r_{j} \sigma_{\mathrm{pol}}$ [6]. Polygenic superiorities for parents of sex $j$ that produce generation $t$ can be represented in vector form as:

$$
\boldsymbol{S}_{j, t}=\sigma_{j} \boldsymbol{i}_{j, t}
$$

where elements of vector $\boldsymbol{i}_{j, t}$ are the selection intensities, which are direct functions of elements of $\boldsymbol{f}_{j, t}$.

Assuming no linkage between the QTL and polygenes, parents on average pass half their polygenic breeding value on to both $\mathrm{B}$ and $\mathrm{b}$ gametes. The mean polygenic breeding value of $\mathrm{B}$ gametes produced by individuals of sex $j$ that create generation $t+1$ is equal to half the sum of the mean polygenic breeding value of selected individuals of each genotype $k\left(B V_{k, t}+i_{j, k, t} \sigma_{j}\right)$, weighted by the frequency of genotype $k$ among selected parents $\left(v_{k, t} f_{j, k, t}\right)$ and by the proportion of gametes produced by genotype $k$ that carry allele $\mathrm{B}\left(N_{k, 1}\right)$ :

$$
\begin{aligned}
A_{\mathrm{s}, 1, t+1}= & 1 / 2\left\{v_{1, t} f_{\mathrm{s}, 1, t}\left(B V_{1, t}+i_{\mathrm{s}, 1, t} \sigma_{\mathrm{s}}\right)+1 / 2 v_{2, t} f_{\mathrm{s}, 2, t}\left(B V_{2, t}+i_{\mathrm{s}, 2, t} \sigma_{\mathrm{s}}\right)\right. \\
& \left.+1 / 2 v_{3, t} f_{\mathrm{s}, 3, t}\left(B V_{3, t}+i_{\mathrm{s}, 3, t} \sigma_{\mathrm{s}}\right)\right\} \\
& /\left(v_{1, t} f_{\mathrm{s}, 1, t}+1 / 2 v_{2, t} f_{\mathrm{s}, 2, t}+1 / 2 v_{3, t} f_{\mathrm{s}, 3, t}\right) .
\end{aligned}
$$


This equation can be rearranged by using equation (7) to simplify the denominator and equations (2), (3) and (10), to see the contribution of the state variables $p_{j, t}$ and $A_{j, t}$, which after multiplying both sides by $p_{\mathrm{s}, 1, t+1}$ results in:

$$
\begin{aligned}
p_{\mathrm{s}, 1, t+1} & A_{\mathrm{s}, 1, t+1} \\
= & 1 / 2\left\{f_{\mathrm{s}, 1, t}\left(\left(A_{\mathrm{s}, 1, t} p_{\mathrm{s}, 1, t} p_{\mathrm{d}, 1, t}+A_{\mathrm{d}, 1, t} p_{\mathrm{d}, 1, t} p_{\mathrm{s}, 1, t}\right)+p_{\mathrm{s}, 1, t} p_{\mathrm{d}, 1, t} S_{\mathrm{s}, 1, t}\right)\right. \\
& +1 / 2 f_{s, 2, t}\left(\left(A_{\mathrm{s}, 1, t} p_{s, 1, t} p_{\mathrm{d}, 2, t}+A_{\mathrm{d}, 2, t} p_{\mathrm{d}, 2, t} p_{\mathrm{s}, 1, t}\right)+p_{\mathrm{s}, 1, t} p_{\mathrm{d}, 2, t} S_{\mathrm{s}, 2, t}\right. \\
& \left.+1 / 2 f_{\mathrm{s}, 3, t}\left(\left(A_{\mathrm{s}, 2, t} p_{\mathrm{s}, 2, t} p_{\mathrm{d}, 1, t}+A_{\mathrm{d}, 1, t} p_{\mathrm{d}, 1, t} p_{\mathrm{s}, 2, t}\right)+p_{\mathrm{s}, 2, t} p_{\mathrm{d}, 1, t} S_{\mathrm{s}, 3, t}\right)\right\} / Q_{\mathrm{s}} .
\end{aligned}
$$

It is convenient to introduce an alternate state variable related to mean polygenic effects of gametes produced by parents of sex $j: W_{j, k, t}=p_{j, k, t} A_{\mathrm{s}, j, t}$ or in vector notation $\boldsymbol{W}_{j, t}=\boldsymbol{p}_{j, t} \circ \boldsymbol{A}_{j, t}$. The advantage is that $\boldsymbol{W}_{j, t}$ is on the same level of computational hierarchy as the $\boldsymbol{p}_{j, t}$ and can be updated simultaneously. Rearranging equation (13) and introducing vector notation, the equations for the update of the average polygenic breeding values for every $t=0, \ldots, T-1$ and $j=\mathrm{s}, \mathrm{d}$ then are:

$$
\boldsymbol{W}_{j, t+1}=1 / 2 \boldsymbol{N}^{\prime}\left(\boldsymbol{f}_{j, t} \circ\left(\boldsymbol{W}_{\mathrm{s}, t} \otimes \boldsymbol{p}_{\mathrm{d}, t}+\boldsymbol{p}_{\mathrm{s}, t} \otimes \boldsymbol{W}_{\mathrm{d}, t}+\boldsymbol{v}_{t} \circ \boldsymbol{S}_{j, t}\right)\right) / Q_{j} .
$$

\subsubsection{Objective function}

The general objective function to be maximized is a weighted sum of the average genetic value in each generation of the planning horizon, with weight $w_{t}$ for generation $t$ (Fig. 2):

$$
R=\sum_{t=0}^{T} w_{t} G_{t}=\sum_{t=0}^{T} w_{t} \boldsymbol{\nu}_{t}^{\prime} \boldsymbol{g}_{t}=\boldsymbol{w}^{\prime} \boldsymbol{G}
$$

where $\boldsymbol{w}$ is a vector with components $w_{t}$ and $\boldsymbol{G}$ a vector with components $G_{t}$. Weights $w_{t}$ can be chosen on the basis of discount factors: $w_{t}=1 /(1+\rho)^{t}$, where $\rho$ is the interest rate per generation. Alternatively, if the aim is to maximize response at the end of the planning horizon, i.e., terminal response, $w_{t}=0$ for $t=0, \ldots, T-1$, and $w_{t}=1$ for $t=T$.

Objective $R$ can be expressed in terms of the state variables $\boldsymbol{p}_{j, t}$ and $\boldsymbol{W}_{j, t}$ as:

$$
\begin{aligned}
R & =\sum_{t=0}^{T} w_{t}\left(\boldsymbol{p}_{\mathrm{s}, t} \otimes \boldsymbol{p}_{\mathrm{d}, t}\right)^{\prime}\left(\boldsymbol{q}+\boldsymbol{A}_{\mathrm{s}, t} \otimes \boldsymbol{J}_{2}+\boldsymbol{J}_{2} \otimes \boldsymbol{A}_{\mathrm{d}, t}\right) \\
& =\sum_{t=0}^{T} w_{t}\left\{\left(\boldsymbol{p}_{\mathrm{s}, t} \otimes \boldsymbol{p}_{\mathrm{d}, t}\right)^{\prime} \boldsymbol{q}+\boldsymbol{W}_{\mathrm{s}, t}^{\prime} \boldsymbol{J}_{2}+\boldsymbol{W}_{\mathrm{d}, t}^{\prime} \boldsymbol{J}_{2}\right\} .
\end{aligned}
$$

The latter equality follows from substituting $\boldsymbol{W}_{j, t}=\boldsymbol{p}_{j, t} \circ \boldsymbol{A}_{j, t}$. 


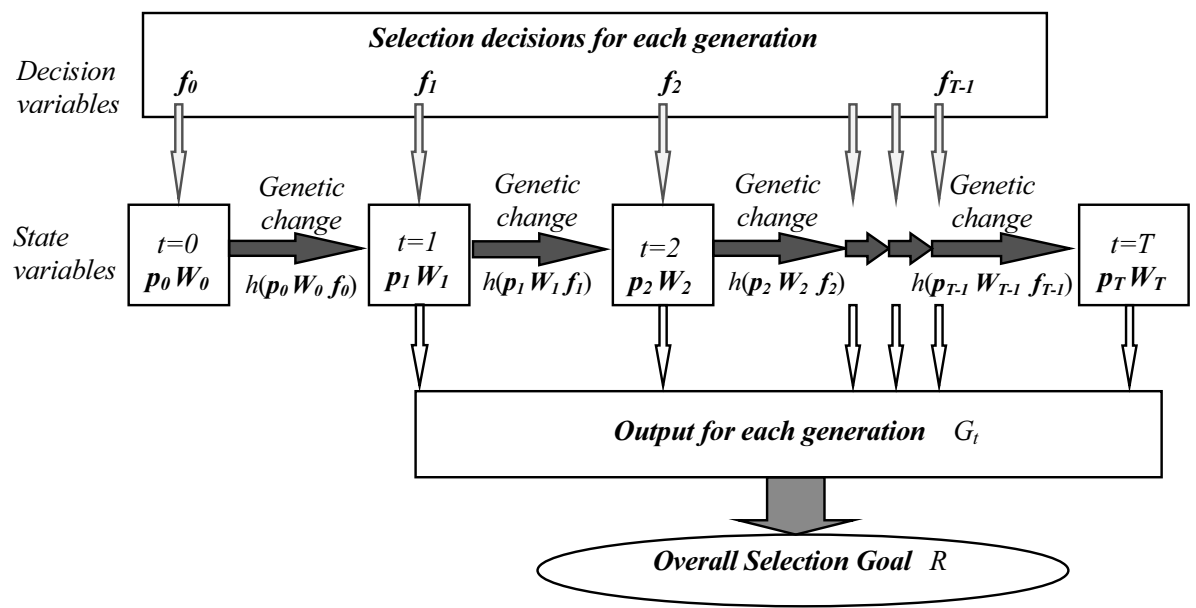

Figure 2. Representation of selection over $T$ generations as a multiple-stage decision problem.

\subsection{Generalization to multiple alleles and multiple QTL}

For the general case of multiple QTL and multiple alleles per QTL, the vector equations developed for one QTL with two alleles still hold, but some variables must be redefined and all vectors and matrices must be properly dimensioned. The main difference is that instead of QTL alleles, the model must be formulated in terms of QTL haplotypes that combine alleles from all identified QTL. For $n q$ QTL with $n a_{q}$ alleles for QTL $q$, the number of possible haplotypes, $n h$, is

$$
n h=\prod_{q=1}^{q=n q} n a_{q} .
$$

Based on modeling at the level of QTL haplotypes instead of alleles, vectors $\boldsymbol{p}_{j, t}$ are redefined as $n h \times 1$ column vectors, the elements of which are frequencies of paternal $(j=\mathrm{s})$ or maternal $(j=\mathrm{d})$ gametes of each haplotype. QTL genotypes are defined by paternal and maternal haplotypes, and the number of possible genotypes, $n g$, is equal to $n h^{2}$. Each vector and matrix that was dimensioned according to the number of alleles and genotypes in the case of one QTL with two alleles, is re-dimensioned accordingly on the basis of the number of haplotypes and multiple QTL genotypes.

Elements of the $n g \times 1$ vector of QTL genotype effects $q$ now represent the total genetic value of each multiple QTL genotype. Note that vector $\boldsymbol{q}$ can accommodate all types of gene action, including epistasis. Because genotypes are distinguished by paternal and maternal haplotypes, vector $\boldsymbol{q}$ can also accommodate gametic imprinting. 
Linkage between identified QTL is accommodated by the $n g \times n h$ matrix $N$, the elements of which correspond to the frequency of each haplotype that is produced by each genotype. As an example, Table II shows the genotypes, genotype frequencies, QTL effects, average breeding values, and the corresponding $N$ matrix for two QTL with recombination rate $r$, two alleles per QTL, and no epistasis.

\subsection{The optimization problem}

Based on the previously developed model, the general optimization problem for a planning period of $T$ generations is:

Given parameters in the starting population: $\boldsymbol{p}_{\mathrm{s}, 0}, \boldsymbol{p}_{\mathrm{d}, 0}, \boldsymbol{A}_{\mathrm{s}, 0}, \boldsymbol{A}_{\mathrm{d}, 0}$

$$
\text { maximize: } R=\sum_{t=0}^{T} w_{t} \boldsymbol{v}_{t}^{\prime} \boldsymbol{g}_{t}=\sum_{t=0}^{T} w_{t}\left\{\left(\boldsymbol{p}_{\mathrm{s}, t} \otimes \boldsymbol{p}_{\mathrm{d}, t}\right)^{\prime} \boldsymbol{q}+\boldsymbol{W}_{\mathrm{s}, t}^{\prime} \boldsymbol{J}_{n h}+\boldsymbol{W}_{\mathrm{d}, t}^{\prime} \boldsymbol{J}_{n h}\right\}
$$

subject to, for every $t=0,1, \ldots, T-1$ and $j=\mathrm{s}$, d:

$$
\begin{gathered}
Q_{j}-\boldsymbol{f}_{j, t}^{\prime}\left(\boldsymbol{p}_{\mathrm{s}, t} \otimes \boldsymbol{p}_{\mathrm{d}, t}\right)=0 \\
\boldsymbol{p}_{j, t+1}=\boldsymbol{N}^{\prime}\left(\boldsymbol{f}_{j, t} \circ\left(\boldsymbol{p}_{\mathrm{s}, t} \otimes \boldsymbol{p}_{\mathrm{d}, t}\right)\right) / Q_{j} \\
\boldsymbol{W}_{j, t+1}=1 / 2 \boldsymbol{N}^{\prime}\left(\boldsymbol{f}_{j, t} \circ\left(\boldsymbol{W}_{\mathrm{s}, t} \otimes \boldsymbol{p}_{\mathrm{d}, t}+\boldsymbol{p}_{\mathrm{s}, t} \otimes \boldsymbol{W}_{\mathrm{d}, t}+\left(\boldsymbol{p}_{\mathrm{s}, t} \otimes \boldsymbol{p}_{\mathrm{d}, t}\right) \circ\left(\sigma_{j} \boldsymbol{i}_{j, t}\right)\right)\right) / Q_{j} .
\end{gathered}
$$

Equations (18b) and (18c) correspond to $n h$ equations per sex, one per QTL haplotype. A separate constraint requiring that haplotype frequencies sum to unity for each sex is unnecessary because this constraint is implicit in matrix $N$ (see Appendix A).

Because of the recursive nature of the constraint equations (18b) and (18c), this maximization problem can be solved using optimal control theory $[5,14]$. The approach presented here follows Dekkers and van Arendonk [5], with $\boldsymbol{f}_{j, t}$ as decision variables and $\boldsymbol{p}_{j, t}$ and $\boldsymbol{W}_{j, t}$ as state variables.

First, a Lagrangian objective function is formulated by augmenting the objective function with each of the equality constraints, which converts the constrained optimization problem into an unconstrained optimization problem. Let $\gamma_{\mathrm{s}, t}$ and $\gamma_{\mathrm{d}, t}$ be Lagrange multipliers for the constraints on fractions selected (equations (18a)), $\boldsymbol{\Lambda}_{\mathrm{s}, t}$ and $\boldsymbol{\Lambda}_{\mathrm{d}, t}$ be row vectors of Lagrange multipliers for the haplotype frequency update equations (equations (18b)), and $\boldsymbol{K}_{\mathrm{s}, t}$ and $\boldsymbol{K}_{\mathrm{d}, t}$ be row vectors of Lagrange multipliers for the update equations for polygenic variables $\boldsymbol{W}_{j, t}$ (equations (18c)). The Lagrange multipliers are co-state variables 
Table II. Genotypes, genotype frequencies, QTL effects, mean polygenic breeding values, and elements of matrix $N$ for selection based on two identified bi-allelic QTL with recombination rate $r$. QTL alleles are denoted $A_{1}$ and $A_{2}$ at the first QTL and $B_{1}$ and $B_{2}$ at the second QTL. Additive and dominance allele effects are denoted $a_{\mathrm{A}}$ and $d_{\mathrm{A}}$ for the first QTL and $a_{\mathrm{B}}$ and $d_{\mathrm{B}}$ for the second QTL. Frequencies of QTL haplotypes $A_{1} B_{1}, A_{1} B_{2}, A_{2} B_{1}$ and $A_{2} B_{2}$ are denoted $p_{j, 1, t}, p_{j, 2, t}, p_{j, 3, t}$, and $p_{j, 4, t}$ respectively for $j=\mathrm{s}$, d. Mean polygenic breeding values corresponding to each haplotype, are $A_{j, 1, t}, A_{j, 2, t}, A_{j, 3, t}$, and $A_{j, 4, t}$ respectively for $j=\mathrm{s}$, d.

\begin{tabular}{|c|c|c|c|c|c|c|c|c|}
\hline & & $v_{t}$ & $q$ & $B \boldsymbol{V}_{t}$ & & & & \\
\hline \# & Genotypes & $\begin{array}{l}\text { Genotype } \\
\text { frequencies }\end{array}$ & $\begin{array}{l}\text { QTL } \\
\text { effect }\end{array}$ & $\begin{array}{l}\text { Mean polygenic } \\
\text { breeding value }\end{array}$ & $\begin{array}{c}A_{1} B_{1} \\
\boldsymbol{n}_{1}\end{array}$ & $\begin{array}{c}A_{1} B_{2} \\
\boldsymbol{n}_{2}\end{array}$ & $\begin{array}{c}A_{2} B_{1} \\
n_{3}\end{array}$ & $\begin{array}{c}A_{2} B_{2} \\
n_{4}\end{array}$ \\
\hline 1 & $A_{1} A_{1} B_{1} B_{1}$ & $p_{\mathrm{s}, 1, t} p_{\mathrm{d}, 1, t}$ & $a_{\mathrm{A}}+a_{\mathrm{B}}$ & $A_{\mathrm{s}, 1, t}+A_{\mathrm{d}, 1, t}$ & 1 & 0 & 0 & 0 \\
\hline 2 & $A_{1} A_{1} B_{1} B_{2}$ & $p_{\mathrm{s}, 1, t} p_{\mathrm{d}, 2, t}$ & $a_{\mathrm{A}}+d_{\mathrm{B}}$ & $A_{\mathrm{s}, 1, t}+A_{\mathrm{d}, 2, t}$ & $1 / 2$ & $1 / 2$ & 0 & 0 \\
\hline 3 & $A_{1} A_{2} B_{1} B_{1}$ & $p_{\mathrm{s}, 1, t} p_{\mathrm{d}, 3, t}$ & $d_{\mathrm{A}}+a_{\mathrm{B}}$ & $A_{\mathrm{s}, 1, t}+A_{\mathrm{d}, 3, t}$ & $1 / 2$ & 0 & $1 / 2$ & 0 \\
\hline 4 & $A_{1} A_{2} B_{1} B_{2}$ & $p_{\mathrm{s}, 1, t} p_{\mathrm{d}, 4, t}$ & $d_{\mathrm{A}}+d_{\mathrm{B}}$ & $A_{\mathrm{s}, 1, t}+A_{\mathrm{d}, 4, t}$ & $(1-r) / 2$ & $r / 2$ & $r / 2$ & $(1-r) / 2$ \\
\hline 5 & $A_{1} A_{1} B_{2} B_{1}$ & $p_{\mathrm{s}, 2, t} p_{\mathrm{d}, 1, t}$ & $a_{\mathrm{A}}+d_{\mathrm{B}}$ & $A_{\mathrm{s}, 2, t}+A_{\mathrm{d}, 1, t}$ & $1 / 2$ & $1 / 2$ & 0 & 0 \\
\hline 6 & $A_{1} A_{1} B_{2} B_{2}$ & $p_{\mathrm{s}, 2, t} p_{\mathrm{d}, 2, t}$ & $a_{\mathrm{A}}-a_{\mathrm{B}}$ & $A_{\mathrm{s}, 2, t}+A_{\mathrm{d}, 2, t}$ & 0 & 1 & 0 & 0 \\
\hline 7 & $A_{1} A_{2} B_{2} B_{1}$ & $p_{\mathrm{s}, 2, t} p_{\mathrm{d}, 3, t}$ & $d_{\mathrm{A}}+d_{\mathrm{B}}$ & $A_{\mathrm{s}, 2, t}+A_{\mathrm{d}, 3, t}$ & $r / 2$ & $(1-r) / 2$ & $(1-r) / 2$ & $r / 2$ \\
\hline 8 & $A_{1} A_{2} B_{2} B_{2}$ & $p_{\mathrm{s}, 2, t} p_{\mathrm{d}, 4, t}$ & $d_{\mathrm{A}}-a_{\mathrm{B}}$ & $A_{\mathrm{s}, 2, t}+A_{\mathrm{d}, 4, t}$ & 0 & $1 / 2$ & 0 & $1 / 2$ \\
\hline 9 & $A_{2} A_{1} B_{1} B_{1}$ & $p_{\mathrm{s}, 3, t} p_{\mathrm{d}, 1, t}$ & $d_{\mathrm{A}}+a_{\mathrm{B}}$ & $A_{\mathrm{s}, 3, t}+A_{\mathrm{d}, 1, t}$ & $1 / 2$ & 0 & $1 / 2$ & 0 \\
\hline 10 & $A_{2} A_{1} B_{1} B_{2}$ & $p_{\mathrm{s}, 3, t} p_{\mathrm{d}, 2, t}$ & $d_{\mathrm{A}}+d_{\mathrm{B}}$ & $A_{\mathrm{s}, 3, t}+A_{\mathrm{d}, 2, t}$ & $r / 2$ & $(1-r) / 2$ & $(1-r) / 2$ & $r / 2$ \\
\hline 11 & $A_{2} A_{2} B_{1} B_{1}$ & $p_{\mathrm{s}, 3, t} p_{\mathrm{d}, 3, t}$ & $-a_{\mathrm{A}}+a_{\mathrm{B}}$ & $A_{\mathrm{s}, 3, t}+A_{\mathrm{d}, 3, t}$ & 0 & 0 & 1 & 0 \\
\hline 12 & $A_{2} A_{2} B_{1} B_{2}$ & $p_{\mathrm{s}, 3, t} p_{\mathrm{d}, 4, t}$ & $-a_{\mathrm{A}}+d_{\mathrm{B}}$ & $A_{\mathrm{s}, 3, t}+A_{\mathrm{d}, 4, t}$ & 0 & 0 & $1 / 2$ & $1 / 2$ \\
\hline 13 & $A_{2} A_{1} B_{2} B_{1}$ & $p_{\mathrm{s}, 4, t} p_{\mathrm{d}, 1, t}$ & $d_{\mathrm{A}}+d_{\mathrm{B}}$ & $A_{\mathrm{s}, 4, t}+A_{\mathrm{d}, 1, t}$ & $(1-r) / 2$ & $r / 2$ & $r / 2$ & $(1-r) / 2$ \\
\hline 14 & $A_{2} A_{1} B_{2} B_{2}$ & $p_{\mathrm{s}, 4, t} p_{\mathrm{d}, 2, t}$ & $d_{\mathrm{A}}-a_{\mathrm{B}}$ & $A_{\mathrm{s}, 4, t}+A_{\mathrm{d}, 2, t}$ & 0 & $1 / 2$ & 0 & $1 / 2$ \\
\hline 15 & $A_{2} A_{2} B_{2} B_{1}$ & $p_{\mathrm{s}, 4, t} p_{\mathrm{d}, 3, t}$ & $-a_{\mathrm{A}}+d_{\mathrm{B}}$ & $A_{\mathrm{s}, 4, t}+A_{\mathrm{d}, 3, t}$ & 0 & 0 & $1 / 2$ & $1 / 2$ \\
\hline 16 & $A_{2} A_{2} B_{2} B_{2}$ & $p_{\mathrm{s}, 4, t} p_{\mathrm{d}, 4, t}$ & $-a_{\mathrm{A}}-a_{\mathrm{B}}$ & $A_{\mathrm{s}, 4, t}+A_{\mathrm{d}, 4, t}$ & 0 & 0 & 0 & 1 \\
\hline
\end{tabular}


in the optimization problem. The resulting Lagrangian objective function is:

$$
\begin{aligned}
L= & \sum_{t=0}^{T} w_{t} \boldsymbol{v}_{t}^{\prime} \boldsymbol{g}_{t}-\sum_{t=0}^{T-1}\left\{\gamma_{\mathrm{s}, t}\left[Q_{\mathrm{s}}-\boldsymbol{f}_{\mathrm{s}, t}^{\prime} \boldsymbol{v}_{t}\right]+\gamma_{\mathrm{d}, t}\left[Q_{\mathrm{d}}-\boldsymbol{f}_{\mathrm{d}, t}^{\prime} \boldsymbol{v}_{t}\right]\right. \\
& +\boldsymbol{\Lambda}_{\mathrm{s}, t+1}\left[Q_{\mathrm{s}} \boldsymbol{p}_{\mathrm{s}, t+1}-\boldsymbol{N}^{\prime}\left(\boldsymbol{f}_{\mathrm{s}, t} \circ \boldsymbol{v}_{t}\right)\right]+\boldsymbol{\Lambda}_{\mathrm{d}, t+1}\left[Q_{\mathrm{d}} \boldsymbol{p}_{\mathrm{d}, t+1}-\boldsymbol{N}^{\prime}\left(\boldsymbol{f}_{\mathrm{d}, t} \circ \boldsymbol{v}_{t}\right)\right] \\
& +\boldsymbol{K}_{\mathrm{s}, t+1}\left[Q_{\mathrm{s}} \boldsymbol{W}_{\mathrm{s}, t+1}-1 / 2 \boldsymbol{N}^{\prime}\left(\boldsymbol{f}_{\mathrm{s}, t} \circ\left(\boldsymbol{W}_{\mathrm{s}, t} \otimes \boldsymbol{p}_{\mathrm{d}, t}+\boldsymbol{p}_{\mathrm{s}, t} \otimes \boldsymbol{W}_{\mathrm{d}, t}+\boldsymbol{v}_{t} \circ \boldsymbol{S}_{\mathrm{s}, t}\right)\right)\right] \\
& +\boldsymbol{K}_{\mathrm{d}, t+1} \\
& \left.\times\left[Q_{\mathrm{d}} \boldsymbol{W}_{\mathrm{d}, t+1}-1 / 2 \boldsymbol{N}^{\prime}\left(\boldsymbol{f}_{\mathrm{d}, t} \circ\left(\boldsymbol{W}_{\mathrm{s}, t} \otimes \boldsymbol{p}_{\mathrm{d}, t}+\boldsymbol{p}_{\mathrm{s}, t} \otimes \boldsymbol{W}_{\mathrm{d}, t}+\boldsymbol{v}_{t} \circ \boldsymbol{S}_{\mathrm{d}, t}\right)\right)\right]\right\}
\end{aligned}
$$

where $\boldsymbol{v}_{t}$ has been substituted for $\boldsymbol{p}_{\mathrm{s}, t} \otimes \boldsymbol{p}_{\mathrm{d}, t}$, and $\boldsymbol{S}_{j, t}$ for $\sigma_{j} \boldsymbol{i}_{j}$ to simplify the equation.

Rearranging terms, the Lagrangian objective function can be expressed as:

$$
\begin{aligned}
L= & w_{T} \boldsymbol{v}_{T}^{\prime} \boldsymbol{g}_{T}+\sum_{t=1}^{T-1}\left\{w_{t} \boldsymbol{v}_{t}^{\prime} \boldsymbol{g}_{t}-\gamma_{\mathrm{s}, t}\left[Q_{\mathrm{s}}-\boldsymbol{f}_{\mathrm{s}, t}^{\prime} \boldsymbol{v}_{t}\right]-\gamma_{\mathrm{d}, t}\left[Q_{\mathrm{d}}-\boldsymbol{f}_{\mathrm{d}, t}^{\prime} \boldsymbol{v}_{t}\right]\right. \\
& -\boldsymbol{\Lambda}_{\mathrm{s}, t+1}\left[Q_{\mathrm{s}} \boldsymbol{p}_{\mathrm{s}, t+1}-\boldsymbol{N}^{\prime}\left(\boldsymbol{f}_{\mathrm{s}, t} \circ \boldsymbol{v}_{t}\right)\right]-\boldsymbol{\Lambda}_{\mathrm{d}, t+1}\left[Q_{\mathrm{d}} \boldsymbol{p}_{\mathrm{d}, t+1}-\boldsymbol{N}^{\prime}\left(\boldsymbol{f}_{\mathrm{d}, t} \circ \boldsymbol{v}_{t}\right)\right] \\
& -\boldsymbol{K}_{\mathrm{s}, t+1}\left[Q_{\mathrm{s}} \boldsymbol{W}_{\mathrm{s}, t+1}-1 / 2 \boldsymbol{N}^{\prime}\left(\boldsymbol{f}_{\mathrm{s}, t} \circ\left(\boldsymbol{W}_{\mathrm{s}, t} \otimes \boldsymbol{p}_{\mathrm{d}, t}+\boldsymbol{p}_{\mathrm{s}, t} \otimes \boldsymbol{W}_{\mathrm{d}, t}+\boldsymbol{v}_{t} \circ \boldsymbol{S}_{\mathrm{s}, t}\right)\right]\right. \\
& -\boldsymbol{K}_{\mathrm{d}, t+1} \\
& \left.\times\left[Q_{\mathrm{d}} \boldsymbol{W}_{\mathrm{d}, t+1}-1 / 2 \boldsymbol{N}^{\prime}\left(\boldsymbol{f}_{\mathrm{d}, t} \circ\left(\boldsymbol{W}_{\mathrm{s}, t} \otimes \boldsymbol{p}_{\mathrm{d}, t}+\boldsymbol{p}_{\mathrm{s}, t} \otimes \boldsymbol{W}_{\mathrm{d}, t}+\boldsymbol{v}_{t} \circ \boldsymbol{S}_{\mathrm{d}, t}\right)\right)\right]\right\} .
\end{aligned}
$$

To further simplify subsequent derivations, the stage Hamiltonian [14] $H_{t+1}$ is introduced for animals that will create generation $t+1$, for every $t=0, \ldots, T-1$ :

$$
\begin{aligned}
H_{t+1}= & w_{t}\left(\boldsymbol{v}_{t}^{\prime} \boldsymbol{q}+\boldsymbol{W}_{\mathrm{s}, t}^{\prime} \boldsymbol{J}_{n h}+\boldsymbol{W}_{\mathrm{d}, t}^{\prime} \boldsymbol{J}_{n h}\right)-\left\{\gamma_{\mathrm{s}, t}\left[Q_{\mathrm{s}}-\boldsymbol{f}_{\mathrm{s}, \boldsymbol{t}}^{\prime} \boldsymbol{v}_{t}\right]+\gamma_{\mathrm{d}, t}\left[Q_{\mathrm{d}}-\boldsymbol{f}_{\mathrm{d}, t}^{\prime} \boldsymbol{v}_{t}\right]\right. \\
& +\boldsymbol{\Lambda}_{\mathrm{s}, t+1}\left[Q_{\mathrm{s}} \boldsymbol{p}_{\mathrm{s}, t+1}-\boldsymbol{N}^{\prime}\left(\boldsymbol{f}_{\mathrm{s}, t} \circ \boldsymbol{v}_{t}\right)\right]+\boldsymbol{\Lambda}_{\mathrm{d}, t+1}\left[Q_{\mathrm{d}} \boldsymbol{p}_{\mathrm{d}, t+1}-\boldsymbol{N}^{\prime}\left(\boldsymbol{f}_{\mathrm{d}, t} \circ \boldsymbol{v}_{t}\right)\right] \\
& +\boldsymbol{K}_{\mathrm{s}, t+1} \\
& \times\left[Q_{\mathrm{s}} \boldsymbol{W}_{\mathrm{s}, t+1}-1 / 2 \boldsymbol{N}^{\prime}\left(\boldsymbol{f}_{\mathrm{s}, t} \circ\left(\boldsymbol{W}_{\mathrm{s}, t} \otimes \boldsymbol{p}_{\mathrm{d}, t}+\boldsymbol{p}_{\mathrm{s}, t} \otimes \boldsymbol{W}_{\mathrm{d}, t}+\boldsymbol{v}_{t} \circ \boldsymbol{S}_{\mathrm{s}, t}\right)\right)\right] \\
& +\boldsymbol{K}_{\mathrm{d}, t+1} \\
& \left.\times\left[Q_{\mathrm{d}} \boldsymbol{W}_{\mathrm{d}, t+1}-1 / 2 \boldsymbol{N}^{\prime}\left(\boldsymbol{f}_{\mathrm{d}, t} \circ\left(\boldsymbol{W}_{\mathrm{s}, t} \otimes \boldsymbol{p}_{\mathrm{d}, t}+\boldsymbol{p}_{\mathrm{s}, t} \otimes \boldsymbol{W}_{\mathrm{d}, t}+\boldsymbol{v}_{t} \circ \boldsymbol{S}_{\mathrm{d}, t}\right)\right)\right]\right\}
\end{aligned}
$$

noting that $\boldsymbol{v}_{t}^{\prime} \boldsymbol{g}_{t}=\boldsymbol{v}_{t}^{\prime} \boldsymbol{q}+\boldsymbol{W}_{\mathrm{s}, \boldsymbol{t}}^{\prime} \boldsymbol{J}_{n h}+\boldsymbol{W}_{\mathrm{d}, t}^{\prime} \boldsymbol{J}_{n h}$ (based on equation (16)). 
Substituting in equation (20) results in

$$
L=w_{T} \boldsymbol{v}_{T}^{\prime} \boldsymbol{g}_{T}+\sum_{t=0}^{T-1} H_{t+1} .
$$

A saddle point of the Lagrangian is determined by deriving the first partial derivatives of the Lagrangian with respect to the decision variables $\left(\boldsymbol{f}_{j, t}\right)$, the state variables $\left(\boldsymbol{p}_{j, t}\right.$ and $\left.\boldsymbol{W}_{j, t}\right)$, and the Lagrange multipliers $\left(\gamma_{j, t}, \boldsymbol{\Lambda}_{j, t}\right.$ and $\left.\boldsymbol{K}_{j, t}\right)$, and equating them to zero for each generation [5]. The partial derivatives of the Lagrangian with respect to each of the Lagrange multipliers yield the corresponding constraints (equations (18a), (18b), and (18c)). Partial derivatives with regard to the remaining variables are derived below.

\subsubsection{Partial derivatives with respect to the decision variables $f_{j, t}$}

At the optimum, the following must hold with respect to the decision variables $\boldsymbol{f}_{j, t}$ for every $t=0, \ldots, T-1$ and $j=\mathrm{s}$, d:

$$
\frac{\partial L}{\partial \boldsymbol{f}_{j, t}}=\frac{\partial H_{t+1}}{\partial \boldsymbol{f}_{j, t}}=0
$$

noting that decision variables for generation $t, \boldsymbol{f}_{j, t}$, appear in the Lagrangian $L$ only through the Hamiltonian for stage $t+1, H_{t+1}$. The following equation results for each $t(t=0, \ldots, T-1)$, as derived in Appendix B:

$$
\gamma_{j, t} \boldsymbol{J}_{n g}+\boldsymbol{N} \boldsymbol{\Lambda}_{j, t+1}^{\prime}+1 / 2\left(\boldsymbol{N} \boldsymbol{K}_{j, t+1}^{\prime}\right) \circ\left(\boldsymbol{B} \boldsymbol{V}_{t}+\sigma_{j} \boldsymbol{X}_{j, t}\right)=0
$$

where $\boldsymbol{X}_{j, t}$ are vectors of standard normal truncation points corresponding to the fractions selected $f_{j, t}$ based on the standard normal distribution theory.

\subsubsection{Partial derivatives with respect to $p_{j, t}$}

Next, the partial derivatives of the Lagrangian with respect to the state variables $\boldsymbol{p}_{j, t}^{\prime}$, are set to zero, for every $t=0,1, \ldots, T-1$, and $j=\mathrm{s}$, d:

$$
\frac{\partial L}{\partial \boldsymbol{p}_{j, t}^{\prime}}=\frac{\partial H_{t}}{\partial \boldsymbol{p}_{j, t}^{\prime}}+\frac{\partial H_{t+1}}{\partial \boldsymbol{p}_{j, t}^{\prime}}=0
$$


These relationships, derived in Appendix B, yield the backward equations for the Lagrange multipliers $\boldsymbol{\Lambda}_{\mathrm{s}, t}$ and $\boldsymbol{\Lambda}_{\mathrm{d}, t}$ :

$$
\begin{aligned}
\boldsymbol{\Lambda}_{\mathrm{s}, t}= & \frac{1}{Q_{\mathrm{s}}}\left\{\left[w_{t} \boldsymbol{q}^{\prime}+\gamma_{\mathrm{s}, t} \boldsymbol{f}_{\mathrm{s}, t}^{\prime}+\gamma_{\mathrm{d}, t} \boldsymbol{f}_{\mathrm{d}, t}^{\prime}\right.\right. \\
& \left.+\left(\boldsymbol{\Lambda}_{\mathrm{s}, t+1} \boldsymbol{N}^{\prime}\right) \circ \boldsymbol{f}_{\mathrm{s}, t}^{\prime}+\left(\boldsymbol{\Lambda}_{\mathrm{d}, t+1} \boldsymbol{N}^{\prime}\right) \circ \boldsymbol{f}_{\mathrm{d}, t}^{\prime}\right]\left(\boldsymbol{I}_{n h} \otimes \boldsymbol{p}_{\mathrm{d}, t}\right) \\
& +1 / 2\left(\boldsymbol{K}_{\mathrm{s}, t+1} \boldsymbol{N}^{\prime}\right)\left[\left(\boldsymbol{I}_{n h} \otimes \boldsymbol{W}_{\mathrm{d}, t}\right) \circ\left(\boldsymbol{f}_{\mathrm{s}, t} \otimes \boldsymbol{J}_{n h}{ }_{n h}\right)\right. \\
& \left.\quad+\left(\boldsymbol{I}_{n h} \otimes \boldsymbol{p}_{\mathrm{d}, t}\right) \circ\left(\left(\boldsymbol{S}_{\mathrm{s}, t} \circ \boldsymbol{f}_{\mathrm{s}, t}\right) \otimes \boldsymbol{J}^{\prime}{ }_{n h}\right)\right] \\
& +1 / 2\left(\boldsymbol{K}_{\mathrm{d}, t+1} \boldsymbol{N}^{\prime}\right)\left[\left(\boldsymbol{I}_{n h} \otimes \boldsymbol{W}_{\mathrm{d}, t}\right) \circ\left(\boldsymbol{f}_{\mathrm{d}, t} \otimes \boldsymbol{J}^{\prime}{ }_{n h}\right)\right. \\
& \left.\left.+\left(\boldsymbol{I}_{n h} \otimes \boldsymbol{p}_{\mathrm{d}, t}\right) \circ\left(\left(\boldsymbol{S}_{\mathrm{d}, t} \circ \boldsymbol{f}_{\mathrm{d}, t}\right) \otimes \boldsymbol{J}_{n h}^{\prime}\right)\right]\right\}
\end{aligned}
$$

where $\boldsymbol{I}_{n h}$ is an identity matrix of dimension $n h$ and,

$$
\begin{aligned}
\boldsymbol{\Lambda}_{\mathrm{d}, t}= & \frac{1}{Q_{\mathrm{d}}}\left\{\left[w_{t} \boldsymbol{q}^{\prime}+\gamma_{\mathrm{s}, t} \boldsymbol{f}_{\mathrm{s}, t}^{\prime}+\gamma_{\mathrm{d}, t}{\boldsymbol{\boldsymbol { f } ^ { \prime } , t}}^{\prime}\right.\right. \\
& \left.+\left(\boldsymbol{\Lambda}_{\mathrm{s}, t+1} \boldsymbol{N}^{\prime}\right) \circ \boldsymbol{f}_{\mathrm{s}, t}^{\prime}+\left(\boldsymbol{\Lambda}_{\mathrm{d}, t+1} \boldsymbol{N}^{\prime}\right) \circ \boldsymbol{f}_{\mathrm{d}, t}^{\prime}\right]\left(\boldsymbol{p}_{\mathrm{s}, t} \otimes \boldsymbol{I}_{n h}\right) \\
& +1 / 2\left(\boldsymbol{K}_{\mathrm{s}, t+1} \boldsymbol{N}^{\prime}\right)\left[\left(\boldsymbol{W}_{\mathrm{s}, t} \otimes \boldsymbol{I}_{n h}\right) \circ\left(\boldsymbol{f}_{\mathrm{s}, t} \otimes \boldsymbol{J}_{n h}{ }_{n h}\right)\right. \\
& \left.+\left(\boldsymbol{p}_{\mathrm{s}, t} \otimes \boldsymbol{I}_{n h}\right) \circ\left(\left(\boldsymbol{S}_{\mathrm{s}, t} \circ \boldsymbol{f}_{\mathrm{s}, t}\right) \otimes \boldsymbol{J}^{\prime}{ }_{n h}\right)\right] \\
& +1 / 2\left(\boldsymbol{K}_{\mathrm{d}, t+1} \boldsymbol{N}^{\prime}\right)\left[\left(\boldsymbol{W}_{\mathrm{s}, t} \otimes \boldsymbol{I}_{n h}\right) \circ\left(\boldsymbol{f}_{\mathrm{d}, t} \otimes \boldsymbol{J}_{n h}^{\prime}\right)\right. \\
& \left.\left.+\left(\boldsymbol{p}_{\mathrm{s}, t} \otimes \boldsymbol{I}_{n h}\right) \circ\left(\left(\boldsymbol{S}_{\mathrm{d}, t} \circ \boldsymbol{f}_{\mathrm{d}, t}\right) \otimes \boldsymbol{J}_{n h}^{\prime}\right)\right]\right\} .
\end{aligned}
$$

\subsubsection{Partial derivatives with respect to $W^{\prime}{ }_{j, t}$}

Finally, with respect to the state variables $\boldsymbol{W}_{j, t}^{\prime}$, the following is true at the optimum for every $t=0,1, \ldots, T-1$, and $j=\mathrm{s}$, d:

$$
\frac{\partial L}{\partial \boldsymbol{W}_{j, t}^{\prime}}=\frac{\partial H_{t}}{\partial \boldsymbol{W}_{j, t}^{\prime}}+\frac{\partial H_{t+1}}{\partial \boldsymbol{W}_{j, t}^{\prime}}=0 .
$$

The following backward equations for $\boldsymbol{K}_{\mathrm{s}, t}$ and $\boldsymbol{K}_{\mathrm{d}, t}$ result (Appendix B):

$$
\begin{aligned}
\boldsymbol{K}_{\mathrm{s}, t}=\frac{w_{t}}{Q_{\mathrm{s}}} \boldsymbol{J}_{n h}^{\prime}+\frac{1}{2 Q_{\mathrm{s}}}\left\{\left(\boldsymbol{K}_{\mathrm{s}, t+1} \boldsymbol{N}^{\prime}\right)\left[\left(\boldsymbol{I}_{n h} \otimes \boldsymbol{p}_{\mathrm{d}, t}\right) \circ\left(\boldsymbol{f}_{\mathrm{s}, t} \otimes \boldsymbol{J}^{\prime}{ }_{n h}\right)\right]\right. \\
\left.+\left(\boldsymbol{K}_{\mathrm{d}, t+1} \boldsymbol{N}^{\prime}\right)\left[\left(\boldsymbol{I}_{n h} \otimes \boldsymbol{p}_{\mathrm{d}, t}\right) \circ\left(\boldsymbol{f}_{\mathrm{d}, t} \otimes \boldsymbol{J}^{\prime}{ }_{n h}\right)\right]\right\}
\end{aligned}
$$

and

$$
\begin{aligned}
\boldsymbol{K}_{\mathrm{d}, t}=\frac{w_{t}}{Q_{\mathrm{d}}} \boldsymbol{J}_{n h}^{\prime}+\frac{1}{2 Q_{\mathrm{d}}}\left\{\left(\boldsymbol{K}_{\mathrm{s}, t+1} \boldsymbol{N}^{\prime}\right)\left[\left(\boldsymbol{p}_{\mathrm{s}, t} \otimes \boldsymbol{I}_{n h}\right) \circ\left(\boldsymbol{f}_{\mathrm{s}, t} \otimes \boldsymbol{J}^{\prime}{ }_{n h}\right)\right]\right. \\
\left.+\left(\boldsymbol{K}_{\mathrm{d}, t+1} \boldsymbol{N}^{\prime}\right)\left[\left(\boldsymbol{p}_{\mathrm{s}, t} \otimes \boldsymbol{I}_{n h}\right) \circ\left(\boldsymbol{f}_{\mathrm{d}, t} \otimes \boldsymbol{J}^{\prime}{ }_{n h}\right)\right]\right\} .
\end{aligned}
$$




\subsubsection{Partial derivatives of the Lagrangian at the terminal conditions}

Equations (26), (27), (29) and (30) are true at the optimum for variables for generations $t=0$ to $T-1$. In the terminal generation, $t=T$, partial derivatives of the Lagrangian with respect to the state variables take on a simplified form that yield the so-called terminal conditions that must be satisfied at the optimum. The following equations result:

$$
\frac{\partial L}{\partial \boldsymbol{p}_{j, T}^{\prime}}=\frac{\partial w_{T} G_{T}}{\partial \boldsymbol{p}_{j, T}^{\prime}}+\frac{\partial H_{T}}{\partial \boldsymbol{p}_{j, T}^{\prime}}=0
$$

which gives

$$
\boldsymbol{\Lambda}_{\mathrm{s}, T}=\frac{w_{T}}{Q_{\mathrm{s}}} \boldsymbol{q}^{\prime}\left(\boldsymbol{I}_{n h} \otimes \boldsymbol{p}_{\mathrm{d}, T}\right)
$$

and

$$
\boldsymbol{\Lambda}_{\mathrm{d}, T}=\frac{w_{T}}{Q_{\mathrm{d}}} \boldsymbol{q}^{\prime}\left(\boldsymbol{p}_{\mathrm{s}, T} \otimes \boldsymbol{I}_{n h}\right)
$$

Also,

$$
\frac{\partial L}{\partial \boldsymbol{W}_{j, T}^{\prime}}=\frac{\partial w_{T} G_{T}}{\partial \boldsymbol{W}_{j, T}^{\prime}}+\frac{\partial H_{T}}{\partial \boldsymbol{W}_{j, T}^{\prime}}=0
$$

which gives

$$
\boldsymbol{K}_{j, T} \frac{w_{T}}{Q_{j}} \boldsymbol{J}_{n h}^{\prime} \text { for } j=\mathrm{s}, \mathrm{d}
$$

\subsection{Computational algorithm}

Equations (24), (26), (27), (29), and (30), along with the terminal conditions, equations (32), (33), and (35), and the original constraints equations (18a), (18b), and (18c), form the system of equations that must be solved to obtain the optimal solutions for the fractions to select from each genotype for each sex at each generation $\left(\boldsymbol{f}_{j, t}\right)$. This system forms a so-called two-point boundary value problem [14] that is solved by backward and forward iteration. The "bounds" are the (known) starting values for the population state variables $\left(\boldsymbol{p}_{j, 0}\right.$ and $\left.\boldsymbol{W}_{j, 0}\right)$ and the terminal conditions for the corresponding Lagrange multipliers for the final generation. The system of equations, illustrated in Figure 3, consists of an outer loop of equations with two branches: a forward branch that develops forward in time, from $t=0$ to $T-1$, and updates the state variables $\boldsymbol{p}_{j, t}$ and $\boldsymbol{W}_{j, t}$, and a backward branch of equations that develops backward in time, from $t=T$ to 1 , and updates the corresponding Lagrange multiplier variables equations $\boldsymbol{\Lambda}_{j, t}$ and $\boldsymbol{K}_{j, t}$.

The forward branch requires computation of the decision variables $\boldsymbol{f}_{j, t}$, which are needed to update the state variables $\boldsymbol{p}_{j, t}$ and $\boldsymbol{W}_{j, t}$. Computation of $\boldsymbol{f}_{j, t}$ is 


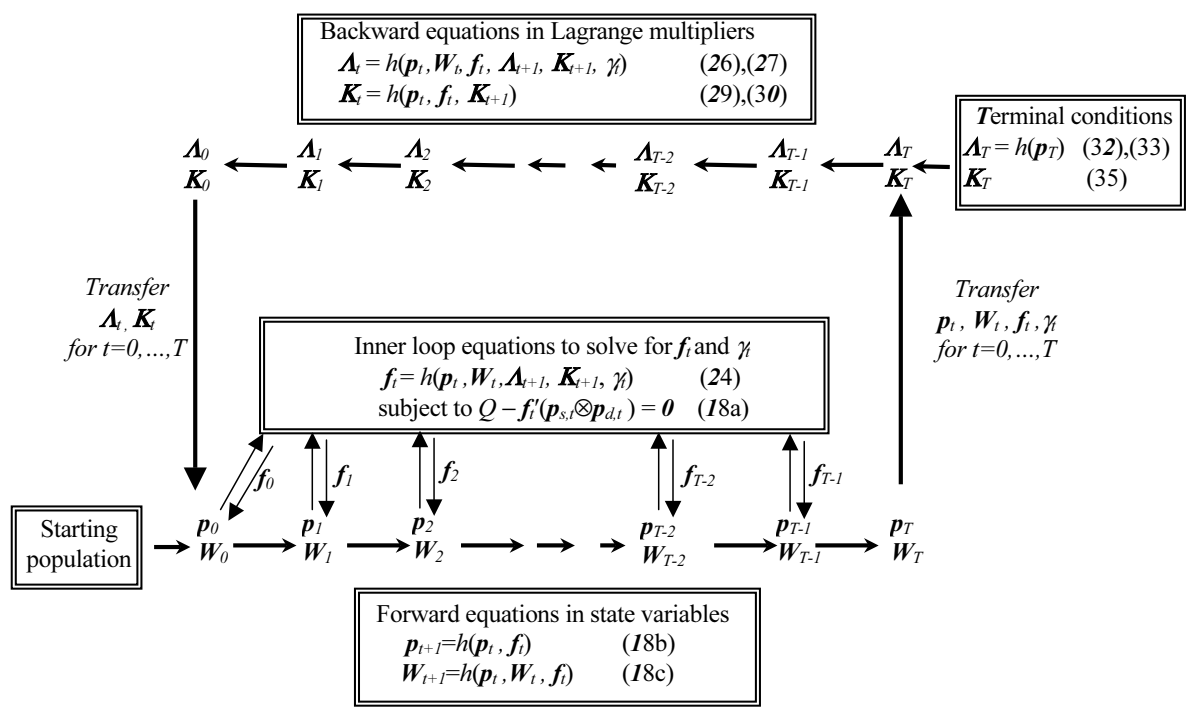

Figure 3. Schematic representation of the two-point boundary problem that results from the optimal control problem and of the iterative procedure for its solution. Numbers in brackets refer to equation numbers in the text. $h(x)$ represents "a function of $x$.

achieved in an inner loop of the forward branch of the outer loop (Fig. 3). This inner loop uses equations (24) and (18a) to compute the truncation points $\boldsymbol{X}_{j, t}$ given the most recently updated values of the Lagrange multipliers $\left(\boldsymbol{\Lambda}_{j, t+1}\right.$ and $\left.\boldsymbol{K}_{j, t+1}\right)$ and the most recently updated values of haplotype frequencies $\left(\boldsymbol{p}_{j, t}\right)$ and mean polygenic breeding values $\left(\boldsymbol{B} \boldsymbol{V}_{t}\right)$, which are obtained from $\boldsymbol{W}_{j, t}$. Given these variables, the truncation points $\boldsymbol{X}_{j, t}$ are solved for by using the bisection algorithm described in Appendix C. This is done separately for each sex $j$ and each generation. Values for the truncation points $\boldsymbol{X}_{j, t}$ are transformed to fractions selected $\left(f_{j, t}\right)$ using the standard normal theory. Updates for the Lagrange multipliers $\gamma_{j, t}$ are simultaneously computed and passed on to the outer loop. Following every pass through the inner loop, from $t=0$ to $T-1$, equations (18b) and (18c) are used to compute updated state variable values for sires and dams for the next generation (i.e. $\boldsymbol{p}_{j, t+1}$ and $\boldsymbol{W}_{j, t+1}$ ). Once the updated values of the state variables are computed for $t=T$, computations cycle to the backward equations.

The backward equations compute updated values for the Lagrange multipliers $\boldsymbol{\Lambda}_{j, t}$ and $\boldsymbol{K}_{j, t}$. This set of the equation is initialized in the terminal generation $\left(\boldsymbol{\Lambda}_{j, t}\right.$ and $\boldsymbol{K}_{j, t}$ ) based on equations (32), (33), and (35) (Fig. 3). Then, proceeding from $t=T-1$ to 1 , new values for the Lagrange multipliers, $\boldsymbol{\Lambda}_{j, t}^{*}$ and $\boldsymbol{K}_{j, t}^{*}$, are computed sequentially via equations (26), (27), (29) and (30), given state and 
decision variables for time $t\left(\boldsymbol{p}_{j, t}, \boldsymbol{W}_{j, t}\right.$, and $\left.\boldsymbol{f}_{j, t}\right)$ and the Lagrange multipliers for generation $t+1\left(\boldsymbol{\Lambda}_{j, t+1}\right.$ and $\left.\boldsymbol{K}_{j, t+1}\right)$.

To enable convergence, relaxation factors $\delta$ are used to limit the step size by which Lagrange multipliers change from one iteration to the next. New values for the Lagrange multipliers are computed as: $\boldsymbol{\Lambda}_{j, t}=\boldsymbol{\Lambda}_{j, t}^{\text {old }}+\delta\left(\boldsymbol{\Lambda}_{j, t}^{*}-\boldsymbol{\Lambda}_{j, t}^{\text {old }}\right)$, where $\boldsymbol{\Lambda}_{j, t}^{\text {old }}$ is the Lagrange multiplier vector from the previous iteration and $\boldsymbol{\Lambda}_{j, t}^{*}$ is the original update. With $\delta=1$, new Lagrange multipliers are accepted as is $\left(\boldsymbol{\Lambda}_{j, t}=\boldsymbol{\Lambda}_{j, t}^{*}\right)$, whereas setting $\delta<1$ reduces the amount of change from one iteration to the next. Similar equations are used for updating the Lagrange multipliers $\boldsymbol{K}_{j, t}$. Experience shows that convergence can be reached in most cases by setting the relaxation factor $\delta$ equal to 0.05 . Ideally, step size would be based on second partial derivatives, as in Newton-Raphson procedures, but this would further complicate derivations.

The objective function is evaluated based on equation (18) after each complete cycle, or iteration, through the outer loop (Fig. 3). The outer loop is iterated until the value of the objective function converges to within a specified tolerance. Although convergence to the global maximum cannot be guaranteed, judicious choice of starting values for the Lagrange multipliers, $\boldsymbol{\Lambda}_{j, t+1}$ and $\boldsymbol{K}_{j, t+1}$, will promote reaching the global maximum. Haplotype frequencies and polygenic means obtained with standard QTL selection can be used to compute such starting values.

\section{RESULTS AND DISCUSSION}

In this paper, a method was developed to optimize selection on multiple identified QTL over multiple generations. The method is general in that it allows for multiple QTL, for arbitrary genetic effects at the identified QTL, including dominance, epistasis, and gametic imprinting, as well as linkage between the identified QTL. A numerical example of the application of the method is in a companion paper [4].

A key ingredient of the model is matrix $N$, which describes the generation of QTL haplotypes from QTL genotypes during meiosis. This matrix is an extension of the transmission matrix that is used in, e.g., segregation analysis. The example presented in Table II illustrates how elements of $\boldsymbol{N}$ accommodate linkage between QTL. The example is for two QTL but a general method has been developed to derive matrix $N$ for an arbitrary number of QTL with any type of linkage between QTL. A description of this method is available from the authors upon request.

The iterative approach developed for solution of the optimization problem capitalizes on the recursive nature of the process of genetic improvement. Specifically, it is recognized that changes in state of the population from the current to the next generation, i.e. changes in gene frequencies and polygenic means, 
depend only on the current state of the population and on the selection decisions made in the current generation, but not on how the population reached the current state. This is a general property of Mendelian inheritance and is reflected in the recursive nature of the equations for QTL frequency (7) and polygenic response (12). Using the optimal control theory, this property is capitalized on through the outer loop of the solution process, by sequentially updating variables using recursive equations (Fig. 3). This recursive process allows for very efficient solution of the optimization problem. In principle this optimization problem can also be solved using more general non-linear programming methods. For example, Manfredi et al. [15] used sequential quadratic programming to solve a related problem. Genetic algorithms have also successfully been applied to the model of Dekkers and van Arendonk [5] (J. van der Werf, personal communication). None of these methods capitalize, however, on the recursive structure of the equations and will, therefore, require substantially more computing time and limit convergence. An advantage of such methods is, however, that they are more flexible with regards to inclusion of additional constraints.

The computational efficiency of the method developed herein will enable its application to a large number of situations and alternatives. Dekkers and Chakraborty [3] recently applied the method to optimal selection with a single QTL for a wide range of additive and dominance effects at the QTL and QTL frequencies. In the example that is reported on in a companion paper [4], the method was applied to optimization of selection on two unlinked or linked QTL with two alleles over ten generations. Derivation of the optimal selection strategy for this case involved optimization of 300 decision variables, i.e. fractions selected for 15 QTL genotypes per sex per generation (the selected fraction for the 16th genotype is obtained by the difference). Convergence of the objective function to within 0.001 was achieved in 300 iterations of the outer loop, which took less than $31 \mathrm{~s}$ of CPU time on a Pentium III processor running at $333 \mathrm{MHz}$. Although the model can in principle handle any number of QTL, convergence issues may be encountered when many QTL are included. Convergence can be enhanced by changing the relaxation factor $\delta$.

Although the method developed here allows for optimization of selection on QTL for a wide range of situations, it is based on several assumptions, which will be discussed in the following paragraphs. Firstly, effects at the QTL are assumed known without error, as are polygenic means by the QTL haplotype. With sufficient population size and a limited number of haplotypes, these parameters should be estimable with sufficient accuracy to make this assumption valid, but its impacts must be validated for other situations. An associated assumption is that QTL genotypes are known. This will be valid for QTL for which the causative mutation is known and approximately valid for QTL that are in strong gametic phase disequilibrium with a single marker or a marker haplotype. 
Another important assumption of the model is that polygenic variance remains constant. Gametic phase disequilibrium among polygenes and changes in allele frequencies at polygenes will invalidate this assumption. If QTL selection is implemented in an ongoing breeding program, however, the impact of gametic phase disequilibrium among polygenes on polygenic variance may be limited. In addition, if the polygenic effect is indeed composed of many genes of small effect, changes in gene frequencies will also be limited.

The present model also assumes that parental origin of QTL alleles can be determined with certainty. Even if parents are genotyped, this will not always be possible, specifically for cases where both parents have the same heterozygous genotype. Unless polygenic means differ substantially between maternal and paternal gametes, the impact of this assumption will, however, be limited for unlinked QTL. Linkage between QTL may increase the impact of not knowing the QTL linkage phase.

It may not be possible to relax the fore-mentioned assumptions in the deterministic model without complicating its optimization. However, the impact of the assumptions can be assessed using stochastic simulation by evaluating the performance of the optimal selection strategies, as derived from the assumed deterministic model, under alternative scenarios and genetic models. Such evaluation is currently underway and preliminary data show that the optimal strategy is rather robust to underlying assumptions. Detailed results will be presented in subsequent papers.

The breeding program modeled here assumed random mating and discrete generations. In principle, the method could be extended to allow for overlapping generations. Allowance for non-random mating will require substantial modification because polygenic effects are modeled at the gametic level. In addition, several additional decision variables would need to be included, specifically mating ratios between alternative genotypes.

The method yields optimal fractions to select from each genotype in each generation of the planning period. In principle, these selection variables can be transformed to weights in a selection index, as was done by Dekkers and van Arendonk [5] for the case of one bi-allelic QTL. The resulting index could be of the following form:

$$
I_{i j m t}=b_{j m t} \theta_{m t}+\left(\widehat{B V}_{i j m t}-B V_{m t}\right)
$$

where $b_{j m t}$ is the weight given to individuals of sex $j$ and QTL genotype $m$ in generation $t, \theta_{m t}$ is the mean breeding value of individuals with QTL genotype $m$ in generation $t$, and $\widehat{B V}_{i j m t}$ is the individual's polygenic breeding value estimate. In the index, $\widehat{B V}_{i j m t}$ is deviated from the mean polygenic breeding value of genotype class $m\left(B V_{m t}\right)$. Note that the QTL genotype here includes all identified QTL and is defined by a combination of paternal and maternal 
QTL haplotypes. In the case of multiple bi-allelic QTL, mean breeding values of QTL genotypes would be derived as:

$$
\theta_{m t}=\sum_{q=1}^{q=n q} n_{q} \alpha_{q t}+\left(B V_{m t}-B V_{r t}\right)
$$

where the summation is over all QTL, the indicator variable $n_{q}$ is equal to -1 , 0 , or 1 for individuals with 0,1 , and 2 favorable alleles at QTL $q, \alpha_{q}$ is the allele substitution effect for QTL $q$ in generation $t\left(\alpha_{q t}=a_{q}+\left(1-2 p_{q t}\right) d_{q}\right.$ where $a_{q}$ and $d_{q}$ are the additive and dominance effects for QTL $q$, and $p_{q t}$ is the frequency of the favorable allele in generation $t$ ), and $B V_{r t}$ is the mean polygenic breeding value for a reference QTL genotype in generation $t$. Following Dekkers and van Arendonk [5], index weights can then be derived for each QTL genotype based on the deviation of its optimal truncation point relative to the reference genotype as: $b_{j m t}=\sigma_{j}\left(X_{j m t}-X_{j r t}\right) / \theta_{m t}$.

For a population of finite size, an optimal selection strategy that is formulated on the basis of index weights will result in different selection decisions than a strategy that is formulated on the basis of fractions selected. Stochastic simulation is needed to determine which implementation results in greater average response to selection.

\section{ACKNOWLEDGEMENTS}

Financial support from the Pig Improvement Company and the NRI Competitive Grants Program/USDA (award no. 98-35205-6736) is greatly appreciated. This is Journal Paper No. J-19215 of the Iowa Agriculture and Home Economics Experiment Station, Ames, Iowa, USA (Project No. 3456) and supported by Hatch Act and State of Iowa Funds. During her stay at ISU, L. Moreau was funded by InRA. Contributions to this work from Jing Wang are gratefully acknowledged.

\section{REFERENCES}

[1] Anderson L., Haley C.S., Ellegren H., Knott S.A., Johansson M., Andersson K., Andersson-Eklund L., Edfors-Lilja I., Fredholm M., Hansson I., Hakansson J., Lundstrom K., Genetic mapping of quantitative trait loci for growth and fatness in pigs, Science 263 (1994) 1771-1774.

[2] Bulmer M.G., The Mathematical Theory of Quantitative Genetics, Clarendon Press, Oxford, 1980.

[3] Dekkers J.C.M., Chakraborty R., Potential gain from optimizing multi-generation selection on an identified quantitative trait locus, J. Anim. Sci. 79 (2001) 29752990. 
[4] Dekkers J.C.M., Chakraborty R., Moreau L., Optimal selection on two quantitative trait loci with linkage, Genet. Sel. Evol. 34 (2001) 171-192.

[5] Dekkers J.C.M., Van Arendonk J.A.M., Optimizing selection for quantitative traits with information on an identified locus in outbred populations, Genet. Res. 71 (1998) 257-275.

[6] Falconer D.S., Mackay T.F.C., Introduction to Quantitative Genetics, Longman, Harlow, 1996.

[7] Fernando R.L., Grossman M., Marker-assisted selection using best linear unbiaised prediction, Genet. Sel. Evol. 21 (1989), 467-477.

[8] Fujii J., Otsu K., Zorzato F., de Leon S., Khanna V.K., Weiler J., O’Brien P.J., MacLennan D.H., Identification of a mutation in the porcine ryanodine receptor that is associated with malignant hyperthermia, Science 253 (1991) 448-451.

[9] Georges M., Nielsen D., Mackinnon M., Mishra A., Okimoto R., Pasquino A.T., Sargeant L.S., Sorensen A., Steele M.R., Zhao X., Womack J.E., Hoeschele I., Mapping quantitative trait loci controlling milk production by exploiting progeny testing, Genetics 139 (1995) 907-920.

[10] Gibson J.P., Short-term gain at the expense of long-term response with selection of identified loci, Proc. 5th World Cong. Genet. Appl. Livest. Prod. 21 (1994) 201-204, University of Guelph, Guelph.

[11] Hospital F., Goldringer I., Openshaw S., Efficient marker-based recurrent selection for multiple quantitative trait loci, Genet. Res., Camb. 75 (2000) 357-368.

[12] Israel C., Weller J.I., Estimation of Candidate Gene Effects in Dairy Cattle Populations, J. Dairy Sci. 81 (1998) 1653-1662.

[13] Larzul C., Manfredi E., Elsen J.M., Potential gain from including major gene information in breeding value estimation, Genet. Sel. Evol. 29 (1997) 161-184.

[14] Lewis F.L., Optimal Control, Wiley, New York, 1986.

[15] Manfredi E., Barbieri M., Fournet F., Elsen J.M., A dynamic deterministic model to evaluate breeding strategies under mixed inheritance, Genet. Sel. Evol. 30 (1998) 127-148.

[16] Pong-Wong R., Woolliams J.A., Response to mass selection when an identified major gene is segregating, Genet. Sel. Evol. 30 (1998) 313-337.

[17] Rothschild M.F., Jacobson C., Vaske D., Tuggle C., Wang L., Short T., Eckardt G., Sasaki S., Vincent A., McLaren D., Southwood O., van der Steen H., Mileham A., Plastow G., The estrogen receptor locus is associated with a major gene influencing litter size in pigs, Proc. Natl. Acad. Sci. USA 93 (1996) 201-205.

[18] Searle S.R., Matrix Algebra for Statisticians, Academic Press, New York, 1982.

[19] Soller M., The use of loci associated with quantitative effects in dairy cattle improvement, Anim. Prod. 27 (1978) 133-139.

\section{APPENDIX A}

\section{Demonstration of the implicit constraint on the sum of haplotype} frequencies

$$
p_{j, 1, t+1}+p_{j, 2, t+1}+\cdots+p_{j, n h-1, t+1}+p_{j, n h, t+1}=1
$$


The elements of the $\boldsymbol{N}$ matrix correspond to the fraction of gametes of a particular haplotype that may be produced from the parental genotype. Matrix $N$ has $n h$ columns, corresponding to each possible haplotype, and $n g$ rows, corresponding to each possible parental genotype. The sum of the frequencies of the gametes produced by a given genotype is equal to one. Thus, the row sum for each row of $N$ is equal to 1 . Let $\boldsymbol{J}_{n g}$ be an $n g \times 1$ column vector, each component of which is one. The columns of the $N$ matrix, $\boldsymbol{n}_{1}, \boldsymbol{n}_{2}, \ldots, \boldsymbol{n}_{n h-1}$, are not linearly independent and,

$$
\boldsymbol{n}_{n h}=\boldsymbol{J}_{n g}-\boldsymbol{n}_{1}-\boldsymbol{n}_{2}-\cdots \boldsymbol{n}_{n h-1} .
$$

The update equation for the vector $\boldsymbol{p}_{j, t+1}$ for $j=\mathrm{s}$ and d, is:

$$
Q_{j} p_{j, t+1}=N^{\prime}\left(f_{j, t} \circ v_{t}\right) .
$$

So for the last haplotype, $n h$,

$$
\begin{aligned}
& Q_{j} p_{j, n h, t+1} \\
& \quad=\boldsymbol{n}_{n h}^{\prime}\left(\boldsymbol{f}_{j, t} \circ \boldsymbol{v}_{t}\right) \\
& \quad=\left(\boldsymbol{J}_{n g}-\boldsymbol{n}_{1}-\boldsymbol{n}_{2}-\cdots-\boldsymbol{n}_{n h-1}\right)^{\prime}\left(\boldsymbol{f}_{j, t} \circ \boldsymbol{v}_{t}\right) \\
& \quad=\boldsymbol{J}^{\prime}{ }_{n g}\left(\boldsymbol{f}_{j, t} \circ \boldsymbol{v}_{t}\right)-\boldsymbol{n}^{\prime}{ }_{1}\left(\boldsymbol{f}_{j, t} \circ \boldsymbol{v}_{t}\right)-\boldsymbol{n}_{2}^{\prime}\left(\boldsymbol{f}_{j, t} \circ \boldsymbol{v}_{t}\right)-\cdots-\boldsymbol{n}_{n h-1}^{\prime}\left(\boldsymbol{f}_{j, t} \circ \boldsymbol{v}_{t}\right) .
\end{aligned}
$$

Note that

$$
\boldsymbol{J}_{n g}^{\prime}\left(\boldsymbol{f}_{j, t} \circ \boldsymbol{v}_{t}\right)=\boldsymbol{f}_{j, t}^{\prime} \boldsymbol{v}_{t}=Q_{j}
$$

and

$$
Q_{j} p_{j, k, t+1}=\boldsymbol{n}_{k}^{\prime}\left(\boldsymbol{f}_{j, t} \circ \boldsymbol{v}_{t}\right) \quad \text { for every } k=1,2, \ldots, n h-1 .
$$

Substituting (A.4) and (A.5) in (A.3), the following is obtained:

$$
Q_{j} p_{j, n h, t+1}=Q_{j}-Q_{j} p_{j, 1, t+1}-Q_{j} p_{j, 2, t+1}-\cdots-Q_{j} p_{j, n h-1, t+1}
$$

which gives the desired result after dividing through by $Q_{j}$,

$$
p_{j, n h, t+1}=1-p_{j, 1, t+1}-p_{j, 2, t+1}-\cdots-p_{j, n h-1, t+1} .
$$

\section{APPENDIX B}

\section{Derivation of partial derivatives of the Lagrangian}

\section{Partial derivatives with regard to $f_{j, t}$}

For the decision variables, for every $t=0, \ldots, T-1$ and $j=\mathrm{s}, \mathrm{d}$, the first derivative of the Lagrangian (equation (23)) with respect to $\boldsymbol{f}_{j, t}$ is:

$$
\frac{\partial L}{\partial \boldsymbol{f}_{j, t}}=\frac{\partial H_{t+1}}{\partial \boldsymbol{f}_{j, t}}=0 .
$$


Referring to the expression for $H_{t+1}$ (equation (19)), note that the term

$$
\boldsymbol{W}_{\mathrm{s}, t} \otimes \boldsymbol{p}_{\mathrm{d}, t}+\boldsymbol{p}_{\mathrm{s}, t} \otimes \boldsymbol{W}_{\mathrm{d}, t}+\boldsymbol{v}_{t} \circ \boldsymbol{S}_{\mathrm{s}, t}=\boldsymbol{v}_{t} \circ\left(\boldsymbol{B} \boldsymbol{V}_{t}+\boldsymbol{S}_{\mathrm{s}, t}\right)
$$

Then,

$$
\begin{aligned}
\frac{\partial H_{t+1}}{\partial \boldsymbol{f}_{j, t}}=\gamma_{j, t} \boldsymbol{v}_{t}+ & \boldsymbol{N} \boldsymbol{\Lambda}_{j, t+1}^{\prime} \circ \boldsymbol{v}_{t} \\
& +1 / 2\left(\boldsymbol{N} \boldsymbol{K}_{j, t+1}^{\prime}\right) \circ\left\{\boldsymbol{v}_{t} \circ\left(\boldsymbol{B} \boldsymbol{V}_{t}+\boldsymbol{S}_{j, t}+\boldsymbol{f}_{j, t} \circ \frac{\partial \boldsymbol{S}_{j, t}}{\partial \boldsymbol{f}_{j, t}}\right)\right\} .
\end{aligned}
$$

Let $\boldsymbol{X}_{j, t}$ be a vector containing the standard normal truncation points corresponding to the fractions selected $\boldsymbol{f}_{j, t}$. The derivative of $\boldsymbol{S}_{j, t}$ with respect to the $f_{j, t}$ can be obtained using the properties of the normal distribution [5] as:

$$
\boldsymbol{f}_{j, t} \circ \frac{\partial \boldsymbol{S}_{j, t}}{\partial \boldsymbol{f}_{j, t}}=-\boldsymbol{S}_{j, t}+\sigma_{\mathrm{s}} \boldsymbol{X}_{j, t}
$$

Substituting in equation (B.2) gives:

$$
\frac{\partial H_{t+1}}{\partial \boldsymbol{f}_{j, t}}=\gamma_{j, t} \boldsymbol{v}_{t}+\boldsymbol{N} \boldsymbol{\Lambda}_{j, t+1}^{\prime} \circ \boldsymbol{v}_{t}+1 / 2\left(\boldsymbol{N} \boldsymbol{K}_{j, t+1}^{\prime}\right) \circ\left\{\boldsymbol{v}_{t} \circ\left(\boldsymbol{B} \boldsymbol{V}_{t}+\sigma_{\mathrm{s}} \boldsymbol{X}_{j, t}\right)\right\}=0
$$

Dividing out $\boldsymbol{v}_{t}$ results in the following equations for $t=0, \ldots, T-1$ :

$$
\gamma_{j, t} \boldsymbol{J}_{n g}+\boldsymbol{N} \boldsymbol{\Lambda}_{j, t+1}^{\prime}+1 / 2\left(\boldsymbol{N} \boldsymbol{K}_{j, t+1}^{\prime}\right) \circ\left(\boldsymbol{B} \boldsymbol{V}_{t}+\sigma_{\mathrm{s}} \boldsymbol{X}_{j, t}\right)=0
$$

\section{Partial derivatives with regard to $p_{j, t}^{\prime}$}

Setting the partial derivatives of the Lagrangian (equation (22)) with respect to the state variables $\boldsymbol{p}_{j, t}$ equal to zero for every $t=0,1, \ldots, T-1$, and $j=\mathrm{s}, \mathrm{d}$ results in:

$$
\frac{\partial L}{\partial \boldsymbol{p}_{\mathrm{s}, t}^{\prime}}=\frac{\partial H_{t+1}}{\partial \boldsymbol{p}_{\mathrm{s}, t}^{\prime}}+\frac{\partial H_{t}}{\partial \boldsymbol{p}_{\mathrm{s}, t}^{\prime}}=0 .
$$

Based on equation (21):

$$
\frac{\partial H_{t}}{\partial \boldsymbol{p}_{j, t}^{\prime}}=-Q_{j} \boldsymbol{\Lambda}_{j, t}
$$


Using the chain rule where necessary (i.e. $\frac{\partial \boldsymbol{g}\left(\boldsymbol{v}_{t}\right)}{\partial \boldsymbol{p}_{j, t}^{\prime}}=\frac{\partial \boldsymbol{g}\left(\boldsymbol{v}_{t}\right)}{\partial \boldsymbol{v}_{t}^{\prime}} \frac{\partial \boldsymbol{v}_{t}}{\partial \boldsymbol{p}_{j, t}^{\prime}}$ ), the derivative $H_{t+1}$ with respect to the $\boldsymbol{p}_{\mathrm{s}, t}^{\prime}$ based on equation (21) takes the form:

$$
\begin{aligned}
& \frac{\partial H_{t+1}}{\partial \boldsymbol{p}_{s, t}^{\prime}}=\left\{w_{t} \boldsymbol{q}^{\prime}+\gamma_{\mathrm{s}, t} \boldsymbol{f}_{\mathrm{s}, t}^{\prime}+\gamma_{\mathrm{d}, t} \boldsymbol{f}_{\mathrm{d}, t}^{\prime}+\left(\boldsymbol{\Lambda}_{\mathrm{s}, t+1} \boldsymbol{N}^{\prime}\right) \circ \boldsymbol{f}_{\mathrm{s}, t}^{\prime}+\left(\boldsymbol{\Lambda}_{\mathrm{d}, t+1} \boldsymbol{N}^{\prime}\right) \circ \boldsymbol{f}_{\mathrm{d}, t}^{\prime}\right\} \\
& \times \frac{\partial \boldsymbol{v}_{t}}{\partial \boldsymbol{p}_{s, t}^{\prime}} \\
& +1 / 2\left(\boldsymbol{K}_{\mathrm{s}, t+1} \boldsymbol{N}^{\prime}\right)\left\{\left(\boldsymbol{I}_{n h} \otimes \boldsymbol{W}_{\mathrm{d}, t}\right) \circ\left(\boldsymbol{f}_{\mathrm{s}, t} \otimes \boldsymbol{J}_{n h}^{\prime}\right)+\frac{\partial \boldsymbol{v}_{t}}{\partial \boldsymbol{p}_{s, t}^{\prime}} \circ\left(\left(\boldsymbol{S}_{\mathrm{s}, t} \circ \boldsymbol{f}_{\mathrm{s}, t}\right) \otimes \boldsymbol{J}_{n h}^{\prime}\right)\right\} \\
& +1 / 2\left(\boldsymbol{K}_{\mathrm{d}, t+1} \boldsymbol{N}^{\prime}\right)\left\{\left(\boldsymbol{I}_{n h} \otimes \boldsymbol{W}_{\mathrm{d}, t}\right) \circ\left(\boldsymbol{f}_{\mathrm{d}, t} \otimes \boldsymbol{J}_{n h}^{\prime}\right)+\frac{\partial \boldsymbol{v}_{t}}{\partial \boldsymbol{p}_{s, t}^{\prime}} \circ\left(\left(\boldsymbol{S}_{\mathrm{d}, t} \circ \boldsymbol{f}_{\mathrm{d}, t}\right) \otimes \boldsymbol{J}_{n h}^{\prime}\right)\right\} .
\end{aligned}
$$

Using $\frac{\partial \boldsymbol{v}_{t}}{\partial \boldsymbol{p}_{s, t}^{\prime}}=\boldsymbol{I}_{n h} \otimes \boldsymbol{p}_{\mathrm{d}, t}$ and substituting in equation (C.1) gives equation (26). Equation (27) is derived in a similar manner based on $\frac{\partial L}{\partial \boldsymbol{p}_{\mathrm{d}, t}^{\prime}}$ $=\frac{\partial H_{t+1}}{\partial \boldsymbol{p}_{\mathrm{d}, t}^{\prime}}+\frac{\partial H_{t}}{\partial \boldsymbol{p}_{\mathrm{d}, t}^{\prime}}=0$ and $\frac{\partial \boldsymbol{v}_{t}}{\partial \boldsymbol{p}_{\mathrm{d}, t}^{\prime}}=\boldsymbol{p}_{\mathrm{s}, t} \otimes \boldsymbol{I}_{n h}$.

\section{Partial derivatives with respect to the $W_{j, t}^{\prime}$}

Setting the partial derivative of the Lagrangian (equation (22)) with respect to the state variables $\boldsymbol{W}_{j, t}$ equal to zero, the following equations are true at the optimum for every $t=0, \ldots, T-1$ and $j=\mathrm{s}$, d:

$$
\frac{\partial L}{\partial \boldsymbol{W}_{j, t}^{\prime}}=\frac{\partial H_{t}}{\partial \boldsymbol{W}_{j, t}^{\prime}}+\frac{\partial H_{t+1}}{\partial \boldsymbol{W}_{j, t}^{\prime}}=0
$$

Using equation (21) for $H_{t}\left(\frac{\partial H_{t}}{\partial W_{j, t}^{\prime}}=-Q_{j} \boldsymbol{K}_{j, t}\right)$ and for $H_{t+1}$, results in the following expression for $j=\mathrm{s}$ :

$$
\begin{aligned}
-Q_{\mathrm{s}} \boldsymbol{K}_{\mathrm{s}, t}+w_{t} \boldsymbol{J}_{n h}^{\prime}+ & 1 / 2\left(\boldsymbol{K}_{\mathrm{s}, t+1} \boldsymbol{N}^{\prime}\right)\left\{\left(\boldsymbol{I}_{n h} \otimes \boldsymbol{p}_{\mathrm{d}, t}\right) \circ\left(\boldsymbol{f}_{\mathrm{s}, t} \otimes \boldsymbol{J}_{n h}^{\prime}\right)\right\} \\
& +1 / 2\left(\boldsymbol{K}_{\mathrm{d}, t+1} \boldsymbol{N}^{\prime}\right)\left\{\left(\boldsymbol{I}_{n h} \otimes \boldsymbol{p}_{\mathrm{d}, t}\right) \circ\left(\boldsymbol{f}_{\mathrm{d}, t} \otimes \boldsymbol{J}_{n h}^{\prime}\right)\right\}=0 .
\end{aligned}
$$

Rearranging gives the backward equations (29) for $\boldsymbol{K}_{\mathrm{s}, t}$. Backward equations for $\boldsymbol{K}_{\mathrm{d}, t}(30)$ are obtained similarly. 


\section{APPENDIX C}

\section{Computational strategy for solving the Inner loop equations}

Equation (24) can be rearranged to get a system of equations that can be solved for the truncation points in generation $t, \boldsymbol{X}_{j, t}$, given all other variables (i.e. $\boldsymbol{\Lambda}_{j, t+1}, \boldsymbol{K}_{j, t+1}, \boldsymbol{p}_{j, t}$, and $\boldsymbol{B} \boldsymbol{V}_{t}$ ):

$$
\gamma_{j, t} \boldsymbol{J}_{n g}+\boldsymbol{N} \boldsymbol{\Lambda}_{j, t+1}^{\prime}+1 / 2\left(\boldsymbol{N} \boldsymbol{K}_{j, t+1}^{\prime}\right) \circ \boldsymbol{B} \boldsymbol{V}_{t}=-1 / 2 \sigma_{\mathrm{s}}\left(\boldsymbol{N} \boldsymbol{K}_{j, t+1}^{\prime}\right) \circ \boldsymbol{X}_{j, t} .
$$

Let $\boldsymbol{\alpha}_{j, t}=1 / 2 \sigma_{\mathrm{s}}\left(\boldsymbol{N K}_{j, t+1}\right)$ and $\boldsymbol{\beta}_{j, t}=\boldsymbol{N} \boldsymbol{\Lambda}_{j, t+1}^{\prime}+1 / 2\left(\boldsymbol{N} \boldsymbol{K}_{j, t+1}^{\prime}\right) \circ \boldsymbol{B} \boldsymbol{V}_{t}$. Then equation (C.1) becomes:

$$
\gamma_{j, t} \boldsymbol{J}_{n g}+\boldsymbol{\beta}_{j, t}=-\boldsymbol{\alpha}_{j, t} \circ \boldsymbol{X}_{j, t} .
$$

Next, subtract one of the equations (for instance the second one) within the vector notation from each equation to eliminate the Lagrange multiplier $\gamma_{j, t}$. Choice of the second equation is arbitrary and subtracting any other equation would give the same optimal solutions. After dividing element wise by $\boldsymbol{\alpha}_{j, t}$, and rearranging terms the following expression is obtained:

$$
\boldsymbol{X}_{j, t}=x_{j, 2, t} \frac{\alpha_{j, 2, t} \boldsymbol{J}_{n g}}{\boldsymbol{\alpha}_{j, t}}+\frac{\beta_{2, j, t} \boldsymbol{J}_{n g}-\boldsymbol{\beta}_{j, t}}{\boldsymbol{\alpha}_{j, t}}
$$

which expresses the truncation points for each genotype in terms of the truncation point for genotype 2. Based on this equation, choice of a truncation point for genotype $2\left(x_{j, 2, t}\right)$ results in associated truncation points for all other genotypes, which when converted to fractions selected $\left(f_{j, t}\right)$ based on standard normal distribution theory, results in an overall fraction selected based on $\boldsymbol{f}_{j, t}^{\prime} \boldsymbol{\nu}_{t}$ (equation (5)). Deviation of $\boldsymbol{f}_{j, t}^{\prime} \boldsymbol{v}_{t}$ from the desired overall fraction selected $Q_{j}$, based on constraint equation (6), i.e. $\Delta_{j}=Q_{j}-\boldsymbol{f}_{j, t}^{\prime} \boldsymbol{v}_{t}$, determines whether the unique truncation points have been found. Since the quantities involved relate to cumulative distribution functions, the function, $\Delta_{j}=Q_{j}-\boldsymbol{f}_{j, t}^{\prime} \boldsymbol{v}_{t}$, is continuous and increasing everywhere; to the left of the unique truncation point $\Delta_{j}$ is negative and to the right it is positive. The intermediate value theorem guarantees both the existence and the uniqueness of the solution to this set of equations.

Because the equations that are involved are riddled with inflexion points, the locations of which are unknown a priori, a fast Newton-Rhapson type method cannot be used; bisection must be used instead. The bisection method consists of the following steps:

1) Pick an upper and lower bound for $x_{j, 2, t}: x_{j, 2, t}^{U}$ and $x_{j, 2, t}^{L}$. 
2) Compute the mean of the upper and lower truncation points:

$$
x_{j, 2, t}^{M}=\left(x_{j, 2, t}^{U}+x_{j, 2, t}^{L}\right) / 2 .
$$

3) Compute truncation points for the other genotypes $\left(\boldsymbol{X}_{j, t}\right)$ based on equation (C.3) and derive the vector of associated fractions selected $\boldsymbol{f}_{j, t}$ based on the standard normal distribution theory.

4) Compute $\Delta_{j}=Q_{j}-\boldsymbol{f}_{j, t}^{\prime} \boldsymbol{v}_{t}$. If $\Delta_{j}>c$, where $c$ is the convergence criterion (e.g. $\left.c=10^{-5}\right)$ set $x_{j, 2, t}^{L}=x_{j, 2, t}^{M}$ and proceed to step 2). If $\Delta_{j}<-c$ set $x_{j, 2, t}^{U}=x_{j, 2, t}^{M}$ and proceed to step 2. If $-c<\Delta_{j}<c$, convergence is reached. 